\title{
Robustness of Liesegang patterns
}

\author{
Arnd Scheel \\ University of Minnesota \\ School of Mathematics \\ 206 Church St. S.E. \\ Minneapolis, MN 55455, USA
}

\begin{abstract}
We propose a conceptual analysis of stationary reaction-diffusion patterns with geometric spatial scaling laws as observed in Liesegang patterns. We give necessary and sufficient conditions for such patterns to occur in a robust fashion. Main ingredients are a skewproduct structure in the kinetics, caused by irreversible chemical reactions, the existence of localized spikes, and slowly decaying boundary layers. The proofs invoke the analysis of homoclinic orbits in orbit-flip position for the spatial dynamics. In particular, we show that there exists a manifold of initial conditions that do not converge to the equilibrium but to the homoclinic orbit as a set.
\end{abstract}

Corresponding author: Arnd Scheel, scheel@umn.edu

Keywords: Liesegang patterns, homoclinic orbit flip, Turing patterns, pulse interaction 


\section{Contents}

1 Introduction and main results $\quad 3$

1.1 Phenomenology and models . . . . . . . . . . . . . . . . . . . 3

1.2 Our main result - a first sketch . . . . . . . . . . . . . . . . 4

1.3 A conceptual approach: ingredients . . . . . . . . . . . . . . 6

1.3.1 Irreversible reactions and skew product dynamics . . . . . . . . . . 6

1.3 .2 Boundary layers . . . . . . . . . . . . . . . . 7

1.3 .3 Turing spots . . . . . . . . . . . . . . . . 7

1.3.4 Glide-interaction balance . . . . . . . . . . . . . . 8

1.3.5 Non-resonance and spectral gap . . . . . . . . . . . . . . . . . . . 9

1.4 Main result . . . . . . . . . . . . . . . . . . . . . . 9

2 A simple mechanistic model $\quad 11$

3 Boundary layers $\quad 13$

4 Turing spots $\quad 14$

5 Dynamics near reversible orbit flip homoclinics $\quad 16$

5.1 Invariant manifolds of multi-spot patterns f . . . . . . . . . . . 16

5.2 Local normal form . . . . . . . . . . . . . . . . . . . . . . 18

5.3 Global reduction . . . . . . . . . . . . . . . . . . . . 18

5.4 Construction of a Poincaré map . . . . . . . . . . . . . . . . 20

5.5 Analysis of the Poincaré map — scaling and invariant manifolds . . . . . . . . 22

5.6 Existence of unstable manifolds - a graph transform proof . . . . . . . . . . 23

5.7 Proof of Theorem $3 \ldots \ldots \ldots \ldots \ldots \ldots$

5.8 Glide-interaction balance in the spatial Poincaré map . . . . . . . . . . . . . 27

5.9 Non-existence of Liesegang patterns . . . . . . . . . . . . . . . . . . . 28

6 Examples $\quad 29$

6.1 A scalar model . . . . . . . . . . . . . . . . . . . . . . 29

6.2 Boundary layers in $A+B \rightarrow C \ldots \ldots \ldots \ldots \ldots$

6.3 Spikes and layers in precipitation . . . . . . . . . . . . . . 30

$\begin{array}{lll}7 & \text { Summary and outlook } & 31\end{array}$ 


\section{Introduction and main results}

\subsection{Phenomenology and models}

While Turing predicted periodic patterns in 1954, it took almost 40 years to realize these structures experimentally in a chemical reaction. A century before, stable patterned states were observed by Liesegang [19]. Liesegang patterns (or rings) are formed as a result of a simple reaction, typically in gels, when the reaction product precipitates in a recurrent fashion; see Figure 1.1, below. Liesegang patterns can be observed in experimental setups, in numerical
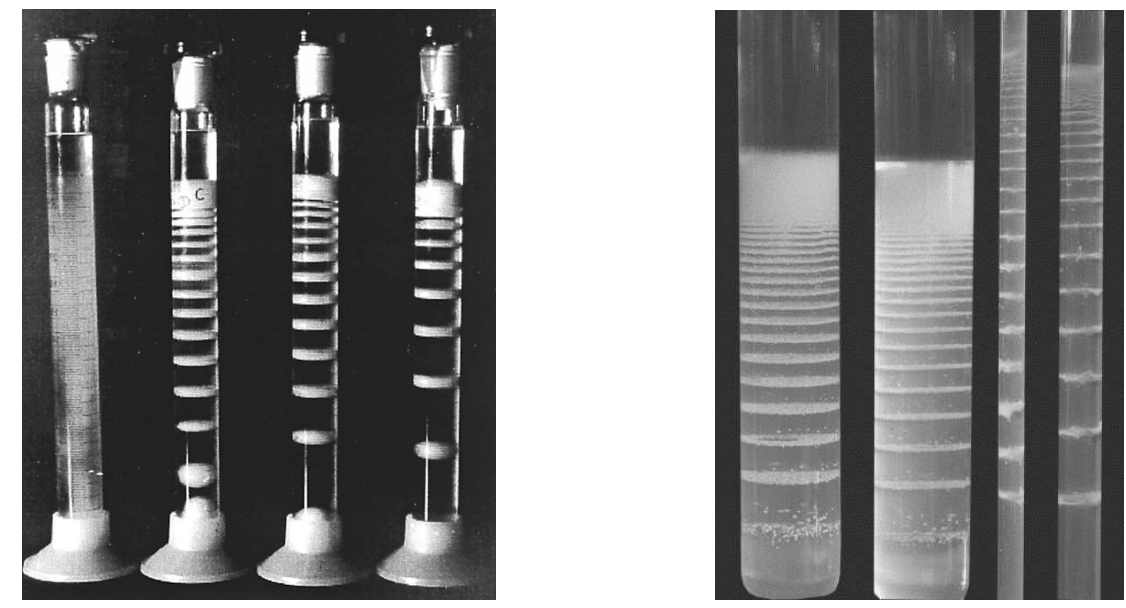

Figure 1.1: Liesegang patterns from [24], left, and [7], right. In the left picture, reactants are $\mathrm{NaOH}$ and $\mathrm{MgCl}_{2}$, with deposit $\mathrm{Mg}(\mathrm{OH})_{2}$. In the right picture, reactants are $\mathrm{H}_{3} \mathrm{PO}_{4}$ and $\mathrm{CaCl}_{2}$ with deposit $\mathrm{Ca}_{3}\left(\mathrm{PO}_{4}\right)_{2}$ (two tubes on the left) and $\mathrm{KF}$ and $\mathrm{Pb}\left(\mathrm{NO}_{3}\right)_{2}$ with deposit $\mathrm{PbF}_{2}$ (two tubes on the right).

simulations, as well as in natural patterns. We refer to $[6,8]$ for recent reviews on experimental and modeling aspects of Liesegang patterns, to [16] for a comparison with natural patterns, and to $[1,5,6,33]$ for potential applications towards a self-organized patterned deposition in the fabrication of materials at the sub-micron scale. In most situations, Liesegang patterns arise as the result of a simple autocatalytic reaction, where two chemical species $A$ and $B$ react to form the product $C$, which then precipitates into a final product $E$. Patterns are formed at the interface of regions where $A$ and $B$ meet and react. One observes that spatially localized deposits of $E$ are formed at distinct locations $x_{i}>0$. The distances $\delta x_{i}=x_{i+1}-x_{i}$ often obey a characteristic spatial scaling law [20],

$$
\delta x_{i+1} / \delta x_{i} \rightarrow \eta, \quad \text { for } i \rightarrow \infty,
$$

where typically $\eta>1$. In addition, one observes a temporal asymptotic scaling law, where the time instance $t_{i}$ of the formation of the deposits $x_{i}$ obeys the scaling $t_{i+1} / t_{i} \rightarrow 2 \eta, \quad$ for $i \rightarrow \infty$, so that the overall spread is diffusive, $x_{i}^{2} / t_{i}$ is asymptotically constant. In many situations, one also observes an increase in width $w_{i}$ of the spatially localized deposits, following roughly an exponential law. However, experimental data on such width laws appears to be varying much stronger than comparable data on distance spacing; see [7], and the discussion in Sections 1.2 and 7 . 
Modeling Liesegang patterns typically involves an irreversible reaction between the chemical species $A$ and $B$, diffusing with diffusivities $d_{A}$ and $d_{B}$, and precipitation kinetics for the products $C$ and $E$. Typical for the precipitation kinetics is a threshold behavior: precipitation is initiated for large $C$ or non-zero $E$, only. Model equations are cast in $x \geq 0$ and are of the form

$$
\begin{aligned}
a_{t} & =d_{a} a_{x x}-a b, & c_{t} & =d_{c} c_{x x}+a b-f(c, e), \\
b_{t} & =d_{b} b_{x x}-a b, & e_{t} & =d_{e} e_{x x}+f(c, e),
\end{aligned}
$$

where $a, b, c, e$ denote the concentrations of the chemical species $A, B, C, E$, respectively, $d_{j}$ are diffusivities, and $f$ models threshold dynamics for the precipitation process; we refer to $[10,14,15,21]$ and $[4,6,9,18]$ for more specific and more general models of Liesegang pattern formation. In the first set of references, the reaction between $A$ and $B$ is assumed to be simply bi-molecular and fast in a certain scaling, while $d_{B}$ is assumed to vanish. Under these assumptions, one finds a diffusive spread of the reactive interface between $A$ and $B$ in the singular limit $[11,12]$. Substituting this diffusive spread into the precipitation kinetics, one obtains temporal scaling laws in the specific precipitation kinetics considered.

In the next section, we outline our approach and sketch our main result and its limitations.

\subsection{Our main result — a first sketch}

In this article, we suggest a complementary, conceptual approach to the understanding of Liesegang patterns, which strives to elucidate the connection between Liesegang and Turing pattern formation. Our approach focuses on the existence of the final Liesegang pattern, that is, a stationary (time-independent) solution to a reaction-diffusion system posed on the positive half line, rather than the successive temporal formation of a finite number of spikes.

The following "Theorem" sketches our main result. The precise result is stated in Theorem 2.

Theorem 1 (Sketch of main result) Consider the class of reaction-diffusion systems $u_{t}=$ $D u_{x x}+f(u) \in \mathbb{R}^{N}$, posed on $x \in \mathbb{R}^{+}$. Assume a skew-product structure induced by an irreversible chemical reaction, separating reactants $u_{1}$ ( $a, b$ in (1.3) and products $u_{2}$ (c,e in (1.3). Then there exists an open set of boundary conditions at $x=0$, reaction kinetics and parameters $f$, and diffusion constants $D$, such that there exists a stationary solution of the reaction-diffusion system, that satisfy the boundary conditions and exhibit a sequence of precipitation spikes spaced at geometrically increasing distances.

The proof is actually constructive: the stationary solution, which we refer to as a Liesegang pattern, is built from two ingredients: we assume that the system would support simple stationary solutions: a stationary boundary layer for the $u_{1}$ part of the system, and a Turing spot (or precipitation spike ${ }^{1}$ for the $u_{2}$-part of the system. The boundary layer is assumed

\footnotetext{
${ }^{1}$ We use the name Turing spot as a place holder for any self-organized, spatially localized, temporally stationary pattern. In a reaction-diffusion system, such a pattern corresponds to a solution $u(x)$ for the system posed on the real line, with $u(x) \rightarrow u_{\infty}$ for $|x| \rightarrow \infty$. In the case of Liesegang patterns, such spots are formed by threshold effects in the precipitation mechanism; see Section 6 .
} 
to be exponentially localized near the boundary and constant elsewhere. The Turing spot is supposed to be a solution on all of $\mathbb{R}$, exponentially localized on a constant background (in fact any such solution would work). We then superimpose the boundary layer and an infinite number of Turing spots and control errors.

Besides these two "simple" stationary solutions, we need a number of technical assumptions, which are satisfied for an open dense set of parameter values. Two assumptions, however appear to be necessary but non-generic:

- the skew-product structure of the kinetics induced by the ireversibility of a chemical reaction; see Section 1.3.1.

- the strong exponential localization of Turing spots (or precipitation spikes) compared to the weak exponential decay of the boundary layer, and a "glide-interaction balance"; see Sections 1.3.4 and 1.4, below.

We remark here that irreversibility is non-generic in the sense that it restricts to kinetics in a subset of possibly high codimension. One may think for instance of setting all reaction constants for reverse reactions to zero. On the other hand, the second set of conditions is merely a sign condition, distinguishing between two open sets of parameter values.

In summary, we give sufficient (and almost necessary) conditions for the existence of Liesegang patterns in a suitable open class of reaction-diffusion systems. As a byproduct, our main result, Theorem 2, predicts asymptotic spatial scaling laws. It also connects Liesegang patterns to multi-spot patterns, consisting of only finitely many spots, and Turing patterns, where spots are spaced equidistantly at $x=\infty$.

Of course, our approach leaves open a number of important questions, some of which will be subject to future work.

- Temporal law. Our approach does not yield any information on the temporal scaling in the formation of Liesegang patterns.

- Stability. An intermediate step towards temporal behavior would be the study of stability of Liesegang patterns with respect to localized, small perturbations in the initial condition. One would expect stable Liesegang patterns to exist whenever boundary layers are stable and interaction of Turing spots is repulsive; see Section 1.3.4 for a definition of Turing spot interaction. We intend to pursue this question further in future work.

- Width law. Our analysis predicts Liesegang patterns with precipitation spikes of fixed width. Conceptually, precipitation spikes are formed by two weakly interacting interfaces, rather than a fixed-width spot. We intend to adapt our analysis to this scenario in future work; see also Section 7

At this stage, our approach does not yield any information on the temporal scaling in the formation of Liesegang patterns. 


\subsection{A conceptual approach: ingredients}

In the following, we specify a number of properties of our reaction-diffusion system that will guarantee the robust occurrence of Liesegang patterns. Our main result, stated in Section 1.4, will make this robustness precise. We will discuss our assumptions and consequences thereof in the following sections. In particular, we will argue that all those assumptions are in some sense necessary ingredients to Liesegang patterns.

We consider reaction-diffusion systems

$$
u_{t}=D u_{x x}+f(u), \quad x \in \mathbb{R}_{+}, u \in \mathbb{R}^{N},
$$

with boundary conditions $B\left(u, u_{x}\right)=0 \in \mathbb{R}^{N}$, at $x=0$. We assume that $D+D^{T}>0$, and that $f: \mathbb{R}^{N} \rightarrow \mathbb{R}^{N}$ and $B: \mathbb{R}^{2 N} \rightarrow \mathbb{R}^{N}$ are smooth. Moreover, we assume that the derivative of $B$ has maximal rank everywhere, so that the zero set of $B$ defines an $N$-dimensional immersed manifold in $\mathbb{R}^{2 N}$. In most experimental scenarios, the diffusion matrix will be diagonal. Much of our analysis would allow some vanishing diffusion coefficients, and we focus on the strictly positive case for ease of notation. Moreover, our considerations are mostly robust in the limit of vanishing diffusivities.

We think of the boundary conditions as mimicking a more complicated interfacial region, where reactants are fed into the domain. In this region, mixing and different domain geometries likely would require a much more complicated setup than the simple one-dimensional reactiondiffusion process, and we therefore choose to absorb this interfacial region into a general nonlinear boundary condition.

In the following, we will make several assumptions on the reaction diffusion system. The assumptions can be thought of as explicit and implicit conditions on $D, f$, and $B$.

\subsubsection{Irreversible reactions and skew product dynamics}

We assume a skew-product structure of the dynamics. More precisely, we assume that $u=$ $\left(u_{1}, u_{2}\right) \in \mathbb{R}^{N_{1}} \times \mathbb{R}^{N_{2}}$, and the reaction diffusion system can be written in the form

$$
\begin{aligned}
& u_{1, t}=D_{1} u_{1, x x}+f_{1}\left(u_{1}\right), \\
& u_{2, t}=D_{2} u_{2, x x}+f_{2}\left(u_{1}, u_{2}\right) .
\end{aligned}
$$

We assume a similar decoupling of the boundary conditions,

$$
B\left(u, u_{x}\right)=\left(B_{1}\left(u_{1}, u_{1, x}\right), B_{2}\left(u, u_{x}\right)\right),
$$

with induced maximal rank conditions on the derivatives of $\left(B_{1}, B_{2}\right)$. The assumption on a decoupling is motivated by the model problems mentioned before. It reflects a more general structural property of irreversibility in chemical reactions. Chemical species in $u_{1}$ may react and form products in $u_{2}$. We however exclude the reversed reaction, where the $u_{1}$-species would be fed from a reaction involving the products $u_{2}$. We will explain later why this type of structural assumption appears to be a necessary condition for the robust occurrence of Liesegang patterns. 


\subsubsection{Boundary layers}

We may consider the subsystem for $u_{1}$ independently. We shall assume that there exists a boundary layer solution $u_{1}^{*}(x), x \geq 0$ to the nonlinear equation with boundary condition, that is,

$$
0=u_{1, x x}^{*}+f_{1}\left(u_{1}^{*}\right), \text { on } x>0 \quad B_{1}\left(u_{1}^{*}(0), u_{1, x}^{*}(0)\right)=0 .
$$

Moreover, we assume that the boundary layer is exponentially localized, a typical assumption as we shall see in Section 3,

$$
u_{1}^{*}(x)=u_{1, \infty}^{*} \mathrm{e}^{-\alpha x}+\mathrm{O}\left(\mathrm{e}^{-(\alpha+\delta) x}\right)
$$

for some vector $u_{1, \infty}^{*}$ and positive constants $\alpha, \delta$. Moreover, we assume that $\alpha$ is the minimal decay rate, that is, there are no non-trivial solutions to $D_{1} u_{1, x x}+f_{1}\left(u_{1}\right)=0$ with $u_{1} \rightarrow 0$ and $\mathrm{e}^{\alpha x} u_{1} \rightarrow \infty$. Lastly, we assume that the linearization at the boundary layer,

$$
\mathcal{L}_{\mathrm{bl}} u=D_{1} u_{x x}+f_{1}^{\prime}\left(u_{1}^{*}\right) u, \quad \partial_{1} B_{1}\left(u_{1}^{*}(0), u_{1, x}^{*}(0)\right) u(0)+\partial_{2} B_{1}\left(u_{1}^{*}(0), u_{1, x}^{*}(0)\right) u_{x}(0)=0,
$$

is invertible.

One can think of the boundary layer acting as an effective parameter ramp in the system for $u_{2}$. Once the boundary layer has formed, the equation for $u_{2}$ is driven by a spatially inhomogeneous forcing term,

$$
u_{2, t}=D_{2} u_{2, x x}+f_{2}\left(u_{1}^{*}(x), u_{2}\right)
$$

\subsubsection{Turing spots}

We next consider the second part of the system in the background state, that is, with $u_{1} \equiv 0$, and $x \in \mathbb{R}$,

$$
u_{2, t}=D_{2} u_{2, x x}+f_{2}\left(0, u_{2}\right) .
$$

We assume that (1.8) possesses a Turing spot solution $u_{2}^{*}(x), x \in \mathbb{R}$ such that $u_{2}^{*}(x)=$ $u_{2}^{*}(-x) \rightarrow 0$ as $|x| \rightarrow \infty$. Moreover we assume that the Turing spot is robust, that is, we assume that the linearization at $u_{2}^{*}$

$$
\mathcal{L} u_{2}:=D_{2} u_{2, x x}+\partial_{2} f_{2}\left(0, u_{2}^{*}\right) u_{2}, \quad x \in \mathbb{R}
$$

is Fredholm of index 0 with an algebraically simple eigenvalue $\lambda=0$, with eigenvector $u_{2, x}^{*}$. We refer to $u_{2}^{*}$ as a Turing spot since it can be viewed as a reaction-diffusion driven, self-organized pattern. Moreover, Turing spots naturally give rise to periodic patterns of large wavelength, which one would refer to as Turing patterns; see Lemma 4.2 .

Besides existence and spectral robustness, we assume monotone tails. More precisely, we assume that $u_{2}^{*}(x)=u_{2, \infty} \mathrm{e}^{-\beta x}+\mathrm{O}\left(\mathrm{e}^{-(\beta+\delta) x}\right)$ as $x \rightarrow \infty$, for some positive constants $\beta, \delta$ and $u_{2, \infty} \in \mathbb{R}^{N_{2}}$. Moreover, we assume that $\beta^{2}$ is simple and the smallest root of $-D_{2}^{-1} f_{2}^{\prime}(0,0)$, in the sense that all other roots $\tilde{\beta}^{2}$ satisfy $|\operatorname{Re} \tilde{\beta}|>|\operatorname{Re} \beta|$. In addition, we assume generic interaction, that is, we assume that $\mathcal{L}$ is invertible in exponentially weighted spaces $L_{\eta}^{2}$ with 
$\eta= \pm(\beta+\delta)$, for $\delta>0$, sufficiently small. As a consequence, we obtain minimal decay for the kernel $\psi$ of the $L^{2}$-adjoint $\mathcal{L}^{*}, \psi(x)= \pm \psi_{\infty} \mathrm{e}^{-\beta|x|}+\mathrm{O}\left(\mathrm{e}^{-(\beta+\delta)|x|}\right)$ as $x \rightarrow \pm \infty$, for some nonzero $\psi_{\infty}$.

The assumption of monotone tails should not be viewed as being restrictive. Oscillatory decay, $\beta \notin \mathbb{R}$, would generically generate a plethora of multi-spot patterns. Intuitively, Turing spots could be placed at arbitrary positions relative to each other such that periodic oscillations in the tails lock, that is, allowed distances vary roughly by multiples of $2 \pi / \operatorname{Im} \beta$. It therefore seems difficult to explain the robust occurrence of the specific geometric scaling in the distances of Liesegang patterns with the assumption of oscillatory decay of Turing spots. On the other hand, multiple eigenvalues $\beta$ or decay with a stronger rate than the minimal eigenvalue should be considered non-generic, so that our assumption covers the generic and interesting case.

\subsubsection{Glide-interaction balance}

The assumption in the previous section guarantees that there is precisely one "slow" degree of freedom associated with each Turing spot, namely the motion by translation. This slow mode is excited by two effects, the effective parameter ramp generated by the boundary layer, and the interaction with neighboring Turing spots. Our last assumption for the existence of Liesegang patterns requires that these two effects carry opposite signs. To be more precise, we normalize the adjoint kernel $\psi \in \operatorname{Ker} \mathcal{L}^{*}$ so that $\int \psi \cdot u_{2, x}^{*}=1$. We say that the boundary conditions are attractive if

$$
F_{\mathrm{bc}}:=\int \psi(x) \cdot \partial_{1} f_{2}\left(0, u_{2}^{*}(x)\right) u_{1, \infty}^{*} \mathrm{e}^{-\alpha x}<0,
$$

and we call the boundary conditions repulsive if $F_{\mathrm{bc}}>0$. We note here that the integrals converge and boundary forces are well-defined only when $\alpha<\beta$; see Remark 1.4.

In a similar fashion, we say that the interaction between Turing spots is attractive if

$$
F_{\text {int }}:=-2 \beta\left(\psi_{\infty}, D u_{2, \infty}\right)<0
$$

and repulsive if $F_{\text {int }}>0$.

We assume that the influence of boundary conditions and interaction can be balanced, that is,

$$
F_{\text {int }} F_{\mathrm{bc}}<0 \text {. }
$$

Remark 1.1 One can formally derive both forces fairly easily by constructing an approximate solution and projecting the error on the kernel associated with an individual Turing spot. Taking for instance a pure Turing spot $u_{2}^{*}$, the error stems from the contribution of the boundary term, which at large values of $x$ is given to leading order by $\partial_{1} f_{2}\left(0, u_{2}^{*}(x)\right) u_{1, \infty}^{*} \mathrm{e}^{-\alpha x}$. This motivates the definition of the boundary force. The interaction force can be explained with the approximate solution $u_{2}(x)=u_{2}^{*}(x-L), x \geq 0, u_{2}(x)=u_{2}^{*}(x+L), x \leq 0$, which creates an error $2 D u_{2, x}^{*}(-L) \delta(x)$. Integrating against the adjoint associated with the spot located at $x=L$ and expanding in L, we find that the induced motion is to the left (reflecting an attractive interaction) for $F_{\text {int }}<0$; see also Section 2. 


\subsubsection{Non-resonance and spectral gap}

In addition to the conceptual assumptions stated above, we will make use of a simplifying assumption with no immediate physical interpretation.

Hypothesis 1.2 (Smooth linearization and reduction) We assume that the eigenvalues of the ODE linearization are non-resonant to order $K$. More precisely, we write $\nu_{j}^{2}, 1 \leq j \leq$ $N$, for the roots of $-D_{1}^{-1} \partial_{1} f_{1}(0,0)$ and $-D_{2}^{-1} \partial_{2} f_{2}(0,0)$, repeated with multiplicity, and we normalize $\operatorname{Re} \nu_{j}>0$. We then assume that $\sum_{j} m_{j} \nu_{j} \neq \nu_{p}$ for any $p$ and any $m \in \mathbb{N}^{N}$ with $2 \leq \sum_{j} m_{j} \leq K$

Moreover, we assume that there is a strong spectral gap between leading and non-leading eigenvalues,

$$
\operatorname{Re} \nu_{j}>2 \beta \text { for all } \nu_{j} \notin\{\alpha, \beta\} \text {. }
$$

We will choose $K$ sufficiently large in our main result. Note however that Hypothesis 1.2 is satisfied for an open set of reaction-diffusion systems for any finite $K$.

We believe that, unlike the previous assumptions, this hypothesis is not necessary for the existence of Liesegang patterns and could be substantially weakened, if not removed altogether. It does however help to simplify proofs considerably.

\subsection{Main result}

In order to state our main result, we give a somewhat general definition of Liesegang-type patterns. We call a stationary solution $u_{\mathrm{ms}}$ an $\varepsilon$-multi-spot pattern if there are locations $x_{j}, 1 \leq j \leq J \leq \infty$, with $x_{0}<x_{1}<x_{2}<\ldots$, so that $u_{\mathrm{ms}}$ is $\varepsilon$-close to a Turing spot on intervals centered at $x_{j}$ and covering $\left\{x \geq x_{0}\right\}$. More precisely, define $x_{j+1}-x_{j}=: \delta x_{j}$, $I_{j}=\left[\left(x_{j}-\delta x_{j-1} / 2, x_{j}+\delta x_{j} / 2\right]\right.$ for $1 \leq j \leq J-1$, and $I_{J}=\left[x_{J}-\delta x_{J-1} / 2, \infty\right)$ if $J<\infty$. We say $u_{\mathrm{ms}}$ is $\varepsilon$-close to Turing spots if

$$
\sup _{I_{j}}\left|u_{\mathrm{ms}, 2}(x)-u_{2}^{*}\left(x-x_{j}\right)\right|<\varepsilon, \quad \text { for } 1 \leq j \leq J .
$$

We then distinguish the following particular cases of multi-spot patterns.

(i) We say $u_{\mathrm{ms}}$ is an $n$-spot pattern if $n=J<\infty$.

(ii) We say $u_{\mathrm{ms}}$ is an asymptotic Turing pattern if $J=\infty$ and $\delta x_{j} \rightarrow 2 \pi / k$ for some wavenumber $k>0$.

(iii) We say $u_{\mathrm{ms}}$ is a Liesegang pattern if $J=\infty$ and $\delta x_{j} \rightarrow \infty$.

In cases (ii) and (iii), we make the definition independent of $\varepsilon$ by requiring that for all $\varepsilon>0$ there exists $x_{0}$ so that $u_{\mathrm{ms}}$ is an $\varepsilon$-multispot pattern. 
Theorem 2 (Robust Liesegang patterns) There is an open nonempty class of reactiondiffusion systems of skew-product type, Section 1.3.1, and boundary conditions (1.6), that possesses stationary Liesegang pattern. More precisely, assume that the reaction-diffusion system exhibits a boundary layer with decay rate $\alpha>0$, Section 1.3.2, Turing spots with decay rate $\beta>\alpha>0$, Section 1.3.3, and that there is a glide-interaction balance, Section 1.3.4. In addition, assume that Hypothesis 1.2 on smooth linearization and reduction holds for some sufficiently large $K$. Then there exists an open non-empty set of boundary conditions such that there exist Liesegang patterns.

Moreover, such Liesegang patterns persist for all nearby boundary conditions $B$, reaction kinetics $f$, and diffusion coefficients $D$, and enjoy the following properties:

- there is a spatial scaling law (1.1) with $\eta=\beta /(\beta-\alpha)$ where $\alpha, \beta$ are decay rates of boundary layer and Turing spot, respectively;

- Liesegang patterns are the locally uniform limit of a family of stationary patterns that exhibit $n$ spots, $n \geq n_{0}$, for any $\varepsilon>0$ with $n_{0}=n_{0}(\varepsilon)$;

- Liesegang patterns depend continuously in $C_{\mathrm{loc}}^{2}$ on parameters in $B, D, f$ that is, they vary continuously as $C^{2}$-functions in any finite window $x \in[0, L]$.

Remark 1.3 The theorem includes two different types of robustness statements. First, it states that given our set of assumptions, we can find an open set of boundary conditions so that there are Liesegang patterns. As one can fairly easily see, the assumptions include a large, open class of reaction-diffusion systems as we will see in the next two sections. The second statement is a local robustness statement: one can vary boundary conditions and system parameters away from values for a particular Liesegang pattern and still find Liesegang patterns, which in addition depend continuously on parameters and boundary conditions. One can easily see that the rate $\eta$ also depends continuously on parameters. Since a small change in $\eta$ implies a large change in the pattern, when measured in a uniform supremum norm, we cannot expect continuous dependence on parameters on the whole real line.

Remark 1.4 The above result is sharp in a certain sense. Neither Liesegang patterns nor finite concatenations of Turing spots exist when

(i) in the absence of a skew-product decoupling, when typically $\alpha=\beta$, see Section 5.9; or

(ii) when the boundary layer is rapidly decaying, $\beta<\alpha$, see Proposition 5.13; or

(iii) when interaction and boundary forces are aligned, $F_{\mathrm{int}} F_{\mathrm{bc}}>0$; see Proposition 5.12.

Outline: In Section 2, we describe a simple, intuitive mechanistic model that illustrates how our assumptions may lead to the existence of Liesegang patterns. Section 3 is concerned with boundary layers in the $u_{1}$-system, their robustness and exponential decay behavior. Section 4 is concerned with Turing spots, their robustness and interaction. Section 5 contains the proof of our main result, which involves an analysis of a homoclinic flip bifurcation. Section 6 shows 
how the results presented here can be applied to some specific reaction-diffusion systems. We conclude with a summary and outlook in Section 7 .

Acknowledgments The author gratefully acknowledges support by the National Science Foundation under grant NSF-DMS-0806614.

\section{A simple mechanistic model}

In this section, we describe a formal reduction of the dynamics of multiple Turing spots on a parameter ramp and how this formal reduction suggests the existence of Liesegang patterns.

First, consider a collection of Turing spots placed at positions $x_{i}$. We construct an almost solution by patching exact Turing spots at their tails, with mismatch in the derivative $u_{2, x}\left(-L_{j}\right)-u_{2, x}\left(L_{j}\right)=2 u_{2, x}\left(-L_{j}\right)$, where $2 L_{j}=x_{j+1}-x_{j}$. The linearization at such an almost-solution possesses an almost kernel spanned by the derivative of each individual spot. Projections on the kernel are obtained by taking scalar products with the translates of the adjoint kernel. Evaluating the mismatch on the right-hand side gives $D \delta\left(x-\left(x_{j+1}-\right.\right.$ $\left.\left.x_{j}\right) / 2\right)\left(u_{2, x}\left(-L_{j}\right)-u_{2, x}\left(L_{j}\right)\right)$. With adjoint kernel given by $\psi\left(x-x_{j}\right)$, we arrive formally at the following effective equation for the positions of Turing spots:

$$
\dot{x}_{j}=2 D u_{2, x}\left(-L_{j}\right) \psi\left(L_{j}\right)+2 D u_{2, x}\left(-L_{j-1}\right) \psi\left(-L_{j-1}\right) .
$$

Substituting the exponential expansion for $u_{2}$ and $\psi$ we arrive at the leading order system

$$
\dot{x}_{j} \sim F_{\text {int }} \cdot\left(\mathrm{e}^{-\beta\left(x_{j}-x_{j-1}\right)}-\mathrm{e}^{-\beta\left(x_{j+1}-x_{j}\right)}\right) .
$$

Since we are interested in patterns with increasing distances, the effective motion is determined by the term coming from the left neighbor,

$$
\dot{x_{j}} \sim F_{\text {int }} \mathrm{e}^{-\beta\left(x_{j}-x_{j-1}\right)} .
$$

In addition to the interaction forces on the right-hand side, we have an effective force created by the boundary condition. Formally projecting the perturbation created by the boundary condition on the kernel gives

$$
\dot{x}_{j} \sim F_{\mathrm{bc}} \mathrm{e}^{-\alpha x_{j}}+F_{\text {int }} \mathrm{e}^{-\beta\left(x_{j}-x_{j-1}\right)} .
$$

In order for the right hand side to vanish, we need precisely the "force balance" sign condition from Section 1.3.4. the resulting dynamics are schematically illustrated in Figure 2. We find equilibria whenever

$$
\alpha x_{j}=\beta\left(x_{j}-x_{j-1}\right)-\log \left(-F_{\text {int }} / F_{\mathrm{bc}}\right) .
$$

From here, we readily derive a geometric Matalon-Packter law $x_{j+1}-x_{j}=\beta /(\beta-\alpha)\left(x_{j}-x_{j-1}\right)$, provided $\beta>\alpha$. For $\alpha>\beta$, we expect that the boundary force cannot be balanced with 

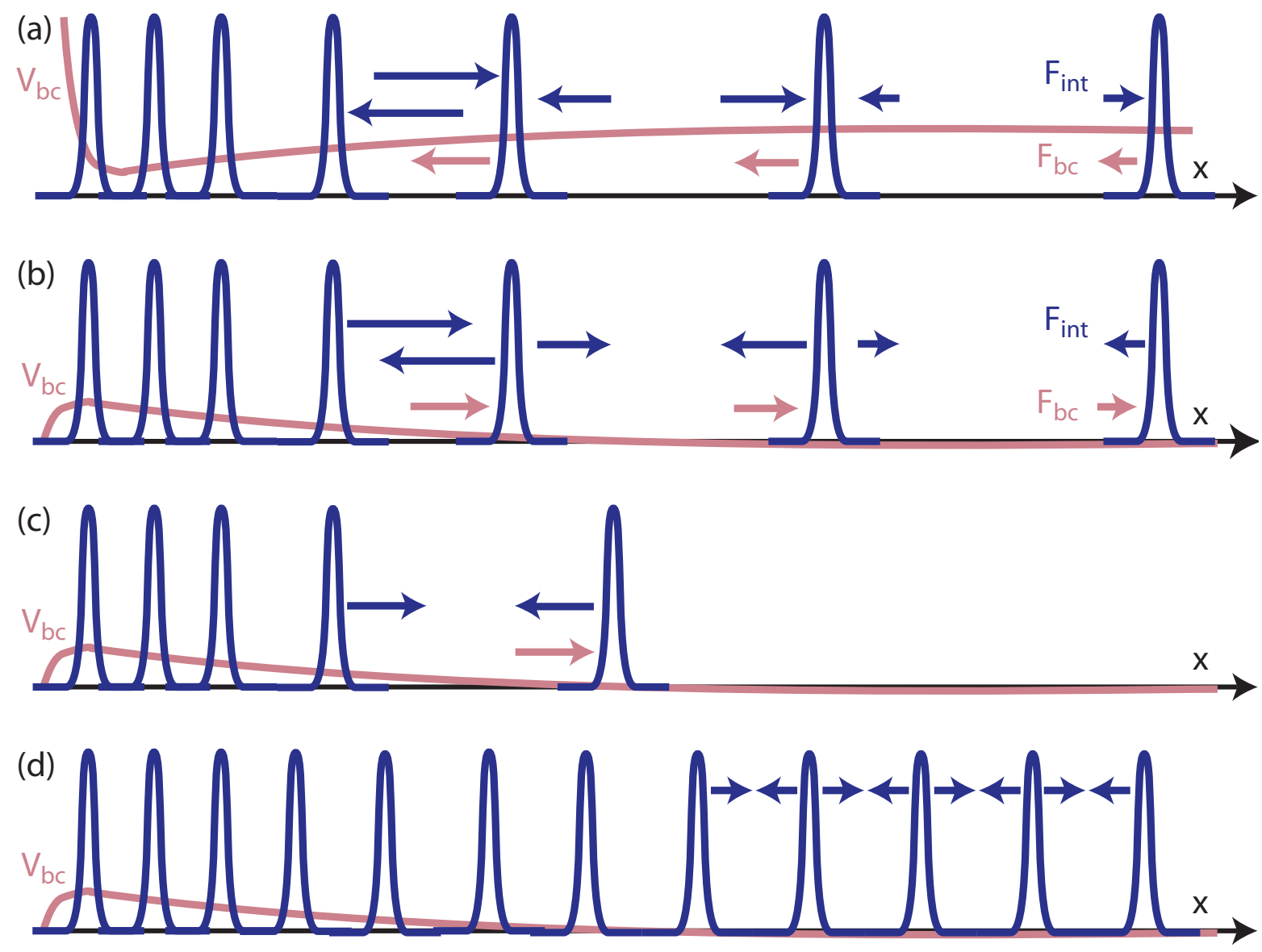

Figure 2.1: A schematic picture of the dynamics of Turing spots under forces $F_{\mathrm{bc}}=-V_{\mathrm{bc}}^{\prime}$, exerted by the boundary conditions, and mutual next-neighbor interaction, $F_{\mathrm{int}}$. We see force balance in a Liesegang pattern with repulsive interaction and attractive boundary in (a). The subsequent cases illustrate attractive interaction and repulsive boundary. They show (b) a Liesegang pattern, (c) a 5spot pattern, and (d) a Turing pattern. Red arrows indicate forces exerted by the boundary conditions, blue arrows indicate interaction forces, and the length of arrows indicates strength of forces.

interaction for large $j, \delta x_{j} \rightarrow \infty$, and (2.1) indeed does not possess stationary solutions with $\delta x_{j} \rightarrow \infty$.

We emphasize that this exact computation is formal in many regards. One can now however "construct" multi-spots, Liesegang patterns, and Turing patterns using only signs and monotonicity of forces. Fix for example a repulsive interaction and an attractive boundary. Placing a single Turing spot at $x_{1} \gg 1$ as initial condition and evolving it with the flow will let the spot move towards the boundary until strong interaction with the boundary stops the motion (a condition on the boundary). Placing a second spot far out would let this second spot approach the first spot until the repulsive interaction balances the attractive force of the boundary condition. One can then "fill in" more and more Turing spots until an infinite number is placed - the Liesegang pattern. On the other hand, one can envision to instantaneously place an infinite number of Turing spots with fixed mutual distance in the medium, 
initially placed at a large distance from the boundary. The left-most spots would then move towards the boundary, while the density at infinity would conceivably remain unchanged by the time evolution.

Summarizing, this construction gives three types of stationary patterns with scaling laws: multi-spot patterns, Liesegang patterns, and Turing patterns. There are however crucial gaps in the argument, resulting from the effective change of the Turing pattern on a parameter ramp which is relaxing at an exponential rate that is slower than the Turing spot. We were not able to give an intuitive understanding for the possibly intricate interaction of those exponentially small effects. As it turns out, our methods will show that the analog and the scaling are correct, although the proof requires a more subtle understanding of the possible behavior in the tails - and, at this point, the additional Hypothesis 1.2.

\section{Boundary layers}

We first concentrate on the patterns generated in the $u_{1}$-subsystem. We will discuss robustness of the assumptions in Section 1.3.1.

We can find stationary boundary layers as solutions to the ordinary differential equation

$$
\begin{aligned}
& u_{1, x}=v_{1} \\
& v_{1, x}=-D_{1}^{-1} f_{1}\left(u_{1}\right) .
\end{aligned}
$$

The boundary conditions define an $N_{1}$-dimensional manifold at $x=0$, which we refer to as $\mathcal{B}_{1}$. We are interested in solutions that converge to zero as $x \rightarrow \infty$. We therefore analyze the linearization at the equilibrium $u_{1}=0$,

$$
u_{1, x}=v_{1}, \quad v_{1, x}=-D_{1}^{-1} f_{1}^{\prime}(0) u_{1},
$$

with eigenvalues $\nu$ given by the square roots of eigenvalues $\rho$ of $-D_{1}^{-1} f_{1}^{\prime}(0)$.

Since we assumed that the linearization at the boundary layer was invertible, hence Fredholm of index zero, there are no bounded solutions to the linearized equation at $\infty$ (these would contribute to essential spectrum at $\lambda=0, \mathcal{L}_{\mathrm{bl}}$ would not be Fredholm) and we can conclude that all eigenvalues $\nu$ are off the imaginary axis. Hypothesis 1.2 actually guarantees that the eigenvalues $\rho$ are ordered $0<\alpha=\nu_{1}<\operatorname{Re} \nu_{j}$, and $\nu_{1}$ is algebraically simple. Our dynamical system (3.1) therefore possesses an $N$-dimensional stable manifold to the origin which we refer to as $W_{1}^{\mathrm{s}}$. Inside of $W_{1}^{\mathrm{s}}$, there is a comdimension-1, $N$-1-dimensional strong stable manifold $W_{1}^{\text {ss }}$, which contains precisely the solutions with strong exponential decay rate, $\mathrm{O}\left(\mathrm{e}^{-(\alpha+\delta) x}\right)$, for some $\delta>0$. Our assumption on the existence of a boundary layer with minimal exponential decay therefore translates into the following geometric statement.

Lemma 3.1 The intersection of $W_{1}^{\mathrm{s}} \backslash W_{1}^{\mathrm{ss}}$ and $\mathcal{B}_{1}$ is nontrivial and contains the boundary layer $u_{1}^{*}$.

The spectral assumption on invertibility of $\mathcal{L}_{\mathrm{bl}}$ ensures that the intersection is transverse and robust. 
Lemma 3.2 Boundary layers with the properties listed occur in open, non-empty classes of reaction-diffusion systems.

Proof. First notice that the intersection of $W_{1}^{\mathrm{s}} \backslash W_{1}^{\text {ss }}$ and $\mathcal{B}_{1}$ is transverse. To see this, notice that a tangency would yield a localized solution to the linearized equation which belongs to the tangent space of the boundary manifold, hence a kernel of $\mathcal{L}_{\mathrm{bl}}$. By smooth dependence of both manifolds and eigenvalue structure on the equation and boundary data, we can conclude that localized boundary layers occur for open classes of reaction-diffusion systems. It remains to show that the class is non-empty. A simple example for a transverse boundary layer is the system $u_{x x}-u=0$, with boundary condition $u=1$ and solution $\mathrm{e}^{-x}$.

\section{Turing spots}

In this section, we discuss our hypothesis on the existence of Turing spots and some consequences. We therefore set $u_{1}=0$ in the system for $u_{2}$ and ignore the boundary conditions at $x=0$ for the time being. The system then can be rewritten as an ordinary differential equation

$$
\begin{aligned}
& u_{2, x}=v_{2} \\
& v_{2, x}=-D_{2}^{-1} f_{2}\left(0, u_{2}\right) .
\end{aligned}
$$

This equation is reversible, that is $\left(u_{2}, v_{2}\right)(x)$ is a solution if and only if $R\left(u_{2}, v_{2}\right)(-x)$ is a solution, where the involution $R$ simply reflects $v, R(u, v)=(u,-v)$. Reversible dynamical systems enjoy a number of properties that are reminiscent of Hamiltonian dynamics (which we would find if the reaction-diffusion system was a - in this context atypical - gradient flow). We refer to $[2,17]$ for reviews of reversible dynamics and more references to the literature.

Our assumption on the existence of a Turing spot $u_{2}^{*}(x)$ guarantees the existence of a homoclinic orbit $\left(u_{2}^{*}, v_{2}^{*}\right) \rightarrow 0$ for $|x| \rightarrow \infty$, of $(4.1)$.

Lemma 4.1 Turing spots with the properties described in Section 1.3.2 occur in open and nonempty classes of reaction-diffusion systems.

Proof. Robustness of Turing spots follows immediately from invertibility of the linearization in spaces of bounded, uniformly continuous, even functions. The single zero-eigenvalue is robust as it is pinned to the origin, generated by translation symmetry. It remains to show robustness of the exponential decay property. Again, we involve the ODE picture. Similarly to the $u_{1}$-subsystem, we find here that $u_{2}=v_{2}=0$ is hyperbolic with stable manifold $W_{2}^{\mathrm{s}}$ and strong stable manifold $W_{2}^{\text {ss }}$. The homoclinic orbit is generated by an intersection of $W_{2}^{\text {s }} \backslash W_{2}^{\text {ss }}$ and the Neumann subspace $v_{2}=0$. The intersection is transverse since the linearization in spaces of even functions is invertible. The intersection therefore depends continuously on perturbations and remains in the open complement of $W_{2}^{\text {ss }}$ inside $W_{2}^{\mathrm{s}}$. A simple example is the localized (albeit unstable) solution to $u_{x x}-u+u^{2}=0$. 
Turing spots are accompanied by long-wavelength Turing patterns.

Lemma 4.2 The homoclinic orbit $q^{*}(x)=\left(u_{2}^{*}(x), v_{2}^{*}(x)\right)$ is accompanied by a unique family of periodic orbits $q_{L}(x):=\left(u_{2}^{L}, v_{2}^{L}\right)(x), L \geq L_{0}$ for some $L_{0}$, with the following properties:

(i) Even: $q_{L}(x)=q_{L}(-x)$;

(ii) Periodic: $q_{L}(x)=q_{L}(x+L)$ for all $x$;

(iii) $q_{L}$ converges to $q^{*}$ in $C_{\text {loc }}^{1}$.

The periodic orbits are stable for repulsive interaction $F_{\text {int }}>0$ and unstable for attractive interaction $F_{\text {int }}<0$.

Proof. The assumptions on the spectrum of the linearization guarantee that the homoclinic is formed by a transverse intersection of the stable manifold of the origin and the reversibility plane $v=0$. We can then apply [34] and conclude the existence of a family of periodic orbits. Stability and instability have been shown in [26, Cor. 5.2].

Combining the dynamic information from the boundary layer and the Turing spots, we find a more complicated dynamical picture. The flow to the ODE

$$
\begin{array}{ll}
u_{1, x}=v_{1} & u_{2, x}=v_{2} \\
v_{1, x}=-D_{1}^{-1} f_{1}\left(u_{1}\right) & v_{2, x}=-D_{2}^{-1} f_{2}\left(u_{1}, u_{2}\right)
\end{array}
$$

possesses an $2 N_{2}$-dimensional invariant subspace given by $u_{1}=v_{1}=0$. Inside this subspace, there is a reversible homoclinic orbit $\left(u_{2}^{*}, u_{2, x}^{*}\right)$, which is accompanied by a family of periodic orbits. The homoclinic orbit is in generic position inside this subspace, that is, stable and unstable manifolds intersect the reversibility (Neumann) subspace $v_{2}=0$ transversely, and the intersection between stable and unstable manifold is of codimension one. The homoclinic approaches the equilibrium along the eigenvector with the weakest decay rate, and the bounded solution to the adjoint equation (which spans the normal to the sum of tangent spaces of stable and unstable manifolds) approaches the adjoint eigenvector to the eigenvalue with the slowest decay rate. This is a structurally stable scenario and homoclinic bifurcation results guarantee that in this typical situation there are no other bounded solutions near the homoclinic orbit.

We are interested in solutions approaching the origin, the homoclinic orbit, or the periodic orbits near the homoclinic. We therefore need to analyze the dynamics in the full $2 N$-dimensional ODE. The main ingredient to dynamics near homoclinic orbits are the eigenvalues near the equilibrium. Our assumption that the eigenvalue $\beta$ inside the $\left(u_{2}, v_{2}\right)$-subspace is larger in modulus than the decay rate of the boundary layer amounts to the statement that the homoclinic orbit is in orbit-flip position, that is, it approaches the origin inside a strong stable manifold, a non-generic scenario for the full $(u, v)$-system; see also Figure 5.1, below. Perturbations that do not respect the skew-product structure of our kinetics would change the decay rate of the homoclinic orbit to $\mathrm{e}^{-\alpha|x|}$ ! 


\section{Dynamics near reversible orbit flip homoclinics}

This section is concerned with orbits of the spatial dynamics (4.2) that correspond to multispot patterns. The main result, Theorem 3, is presented in section 5.1. It describes orbits that stay close to the homoclinic for all forward times. Theorem 2 follows from Theorem 3 in a rather straightforward fashion as we shall see in the remainder of Section 5.1. We shall then proof Theorem 3. The goal is to analyze the flow in a tubular neighborhood of the homoclinic orbit. We therefore first change coordinates in a neighborhood of the equilibrium so that the local flow in this neighborhood is in normal form and actually integrable, Section 5.2 , and then use a global reduction theorem to reduce the flow near the homoclinic to a 3-dimensional invariant manifold, Section 5.3. We next construct a return map to a local Poincaré section near the homoclinic, Section 5.4. The core result is an invariant manifold result for this return map, Section 5.5, which we prove in Section 5.6. Section 5.7 shows how to conclude Theorem 3 from this invariant manifold result on a Poincaré section. Section 5.8 relates the sign of interaction and boundary forces to the sign of a crucial coefficient in the Poincaré map. Section 5.9 discusses cases where Liesegang patterns do not exist, establishing the necessity of some of our assumptions.

\subsection{Invariant manifolds of multi-spot patterns}

In this next step, we analyze the solutions in the first-order differential equation

$$
\begin{array}{ll}
u_{1, x}=v_{1} & u_{2, x}=v_{2} \\
v_{1, x}=-D_{1}^{-1} f_{1}\left(u_{1}\right) & v_{2, x}=-D_{2}^{-1} f_{2}\left(u_{1}, u_{2}\right)
\end{array}
$$

We are interested in solutions that converge towards the homoclinic orbit $\left(u_{1}, v_{1}\right)=0$, $\left(u_{2}, v_{2}\right)=\left(u_{2}^{*}, u_{2, x}^{*}\right)$ in phase space $\mathbb{R}^{2 N}$. We can use the flow to this equation to transport the boundary conditions $\mathcal{B}$ from $x=0$ to $x=x_{0} \gg 1$, so that we can assume that the Liesegang pattern actually is close to a neighborhood of the homoclinic for all $x>0$. The following theorem describes solutions that stay close to the homoclinic orbit for all $x>0$. We will find our main result, Theorem 2 as a simple corollary to this geometric statement on the dynamics near an orbit-flip homoclinic orbit.

Theorem 3 (Liesegang manifold) Suppose that $\alpha<\beta$ and assume force balance. Then there are the following three types of solutions that stay in a sufficiently small neighborhood of the homoclinic for all $x>0$ :

(i) $n$-spot: the solution lies in the stable manifold of the origin and experiences $n$ loops along the homoclinic before remaining in a small neighborhood of the origin; the set of such solutions forms a smooth $N$-dimensional manifold for any $n$ large, arbitrary;

(ii) Turing: the solution converges exponentially to a periodic orbit; the set of such solutions forms a smooth $N$-dimensional manifold for each fixed periodic orbit; 
(iii) Liesegang: the solution converges to the homoclinic as a set; the set of such solutions forms an $N$-dimensional $C^{1}$-manifold with a continuous limit at the equilibrium.

All manifolds depend continuously on parameters. In the last case, the times $T_{n}$ spent between excursions along the homoclinic increase geometrically,

$$
T_{n+1} / T_{n} \rightarrow \frac{\beta}{\beta-\alpha},
$$

The manifold in (iii) is the $C^{1}$-limit of the manifolds in (i) as $n \rightarrow \infty$.

Remark 5.1 It seems plausible that the manifolds in (ii) also limit on the manifold in (iii), that is, that Liesegang patterns are the locally uniform limit of a continuous family of Turing patterns with boundary layers and selected wavenumber $0<k \leq k_{0}$. Investigating this limit of zero-wavenumber Turing patterns however seems to be a more subtle problem.

Figure 5.1, below, shows a schematic picture of the spatial dynamics in phase space that create Liesegang patterns.

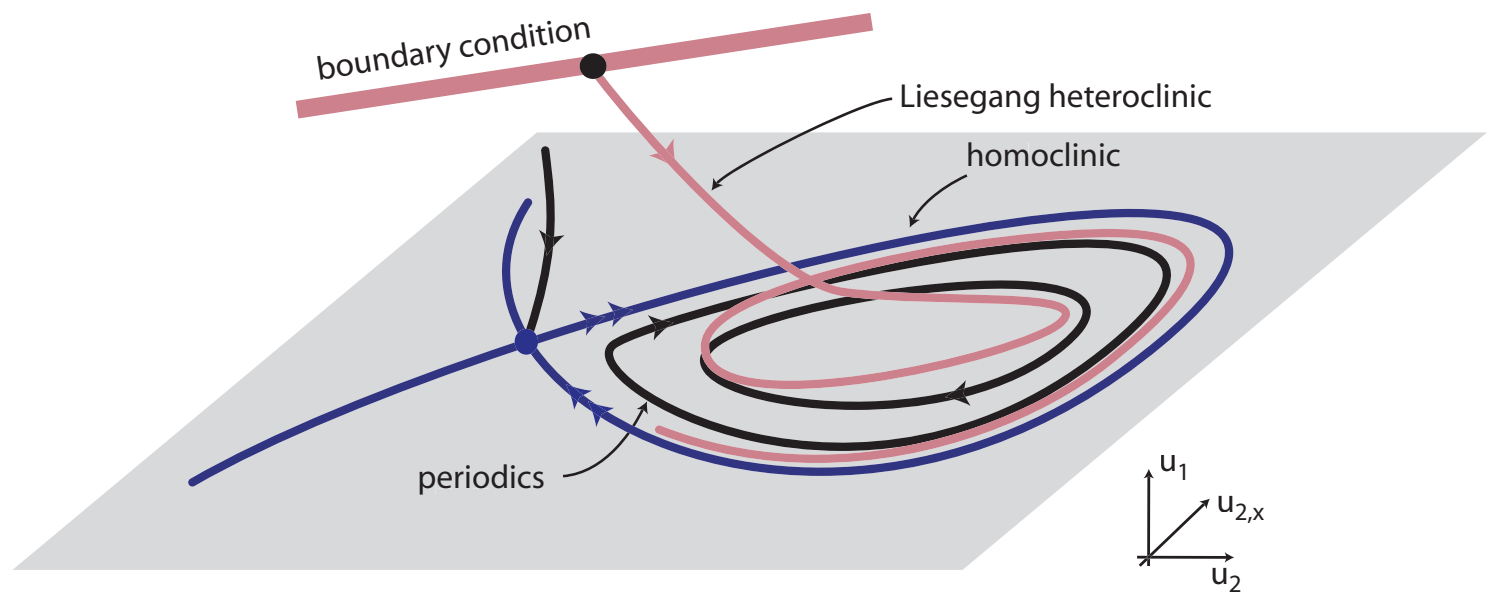

Figure 5.1: A schematic picture of phase space with the invariant $u_{2}$-subspace containing a reversible homoclinic orbit with accompanying periodic orbits, and a Liesegang orbit approaching the homoclinic as a set.

Proof. [Theorem 2] We show that Theorem 3 implies Theorem 2. It is therefore sufficient to choose an $N$-dimensional $C^{1}$-manifold that is

- transverse to the Liesegang manifold in Theorem 3, (iii);

- can be represented in the form $B\left(u, u_{x}\right)=0$.

Indeed, such a transverse intersection would be robust and yield Liesegang patterns as stated in Theorem 2. Of course, transverse manifolds do exist and can be written as the zero set 
of a rank- $N$ function $B$. We need to show however that we can achieve to write $B$ in the specific skew-product form. We therefore write $U_{j}=\left(u_{j}, u_{j, x}\right), j=1,2$, and $U=\left(u, u_{x}\right)$. The Liesegang manifold, projected on the first component $U_{1}$ is simply given by the stable manifold of the origin inside the $u_{1}$-subsystem. We parametrize the tangent space to the Liesegang manifold in the form $U=S w, w \in \mathbb{R}^{N}$ and write $B U=0 \in \mathbb{R}^{N}$ for the linearized boundary conditions. Due to the skew-product structure, we have the specific form

$$
S=\left(\begin{array}{cc}
S_{11} & 0 \\
S_{21} & S_{22}
\end{array}\right), \quad B=\left(\begin{array}{cc}
B_{11} & 0 \\
B_{21} & B_{22}
\end{array}\right), \quad S_{i j}: \mathbb{R}^{N_{j}} \rightarrow \mathbb{R}^{2 N_{i}}, B_{i j}: \mathbb{R}^{2 N_{j}} \rightarrow \mathbb{R}^{N_{i}}
$$

Here $S_{11}$ has the maximal rank $N_{1}$, since it parametrizes the stable manifold in the $U_{1}$-system, and we imposed that $B_{11}$ has rank $N_{1}$. Moreover, $S_{22}$ has rank $N_{2}$ since $S$ has rank $N$. We need to choose $B$ such that $B S$ is invertible on $\mathbb{R}^{N}$, which is equivalent to both $B_{11} S_{11}$ and $B_{22} S_{22}$ being invertible. Choosing $B=B^{0}$ such that the $B_{j j}$ are the orthogonal projections onto the range of $S_{j j}$ is one possible choice that guarantees invertibility. Therefore, $B^{0}\left(U-U_{\text {li }}\right)=0$ defines an affine subspace, which is transverse to the Liesegang manifold.

\subsection{Local normal form}

We first recall the structure of the flow to the system (5.1). The system possesses a homoclinic orbit to the origin, accompanied by periodic orbits in a flow-invariant linear subspace. The linearization at the origin possesses eigenvalues $\pm \alpha, \pm \beta$, and $\pm \nu_{j}$ with $\operatorname{Re} \nu_{j}>\beta>\alpha>0$. Due to the non-resonance conditions, there is a $C^{3}$-change of coordinates such that the vector field in a vicinity of the origin is in Poincaré normal form. More precisely, there are coordinates $\tilde{u}=\Phi(u), \Phi \in C^{3}$ and continuously depending on the vector field, such that the vector field in new coordinates is given by

$$
\tilde{u}_{x}=L \tilde{u}+\tilde{u} P(\tilde{u}),
$$

where $P$ is a vector polynomial function of the resonant monomials $u^{2 j-1} u^{2 j}$, only. The linear part $L$ is (complex) diagonal,

$$
L\left(u^{1}, \ldots, u^{2 N}\right)^{T}=\left(\lambda_{1} u^{1},-\lambda_{1} u^{2}, \ldots, \lambda_{N} u^{2 N-1},-\lambda_{N} u^{2 N}\right)^{T} .
$$

Reversibility acts through $u^{2 j} \mapsto u^{2 j-1}, u^{2 j-1} \mapsto-u^{2 j}$, so that $P$ is actually a constant of motion. The existence of a reversibility-preserving change of coordinates that puts the local vector field into normal form is shown in [28, Thm 2]. Continuous (and even smooth) dependence on parameters can be shown as in [22, 29].

\subsection{Global reduction}

We can restrict the dynamics to the values of $\left(u_{1}, u_{1, x}\right)$ in the stable manifold of the origin inside the $u_{1}$-subsystem. Note that in our local normal form coordinates, this manifold is given by $u^{2 j-1}=0$ for those $j$ corresponding to the first component $u_{1}$. Within this $N_{1}+2 N_{2}$ dimensional manifold, we have the following result on the existence of a 3-dimensional invariant manifold. 
Proposition 5.2 There is $\iota>0$ so that the system (5.1) possesses a 3-dimensional locally invariant manifold $\mathcal{M}_{\mathrm{hom}}$, with the following properties:

- $\mathcal{M}_{\text {hom }}$ contains the homoclinic orbit and all bounded solutions in a neighborhood of the homoclinic;

- $\mathcal{M}_{\mathrm{hom}}$ is $C^{2, \iota}$, diffeomorphic to a solid torus $S^{1} \times B^{2}$ and carries a $C^{2, \iota}$ local flow;

- $\mathcal{M}_{\mathrm{hom}}$ depends on parameters in the system in a $C^{2, \iota}$-fashion;

- $\mathcal{M}_{\text {hom }}$ is normally hyperbolic and possesses a $C^{2, L}$-strong stable foliation, which also depends on parameters in a $C^{2, \iota}$-fashion.

Proof. Existence and smoothness of the center manifold with the first three properties listed are a consequence of the center-manifold theorem in [25]. Existence of smooth foliations can be proved as in [13], as a consequence of normal hyperbolicity and the fact that the manifold is overflowing after a suitable modification of the flow. We need to verify the assumptions of the results in [25]. First, we need to show transversality of certain bundles in the linearization. One therefore constructs the subspaces

$$
\begin{array}{lrl}
E_{+}^{\mathrm{ss}} \text { : solutions with }|u(x)| \leq C \mathrm{e}^{-(\beta+\delta) x}, & x \rightarrow \infty \text { of dimension } N_{1}+N_{2}-2 ; \\
E_{+}^{\mathrm{cs}} \text { : solutions with }|u(x)| \leq C \mathrm{e}^{(\beta+\delta) x}, & x \rightarrow \infty \text { of dimension } N_{1}+N_{2}+1 ; \\
E_{-}^{\mathrm{uu}} \text { : solutions with }|u(x)| \leq C \mathrm{e}^{(\beta+\delta) x}, & x \rightarrow-\infty \text { of dimension } N_{2}-1 ; \\
E_{-}^{\mathrm{cu}} \text { : solutions with }|u(x)| \leq C \mathrm{e}^{-(\beta+\delta) x}, & x \rightarrow-\infty \text { of dimension } N_{2}+2 ;
\end{array}
$$

The assumptions on the existence of a homoclinic center manifold require that the intersections $E_{+}^{\mathrm{ss}} \cap E_{-}^{\mathrm{cu}}$ and $E_{+}^{\mathrm{cs}} \cap E_{-}^{\mathrm{uu}}$ are trivial, that is, the intersections are transverse. First notice that a nontrivial intersection cannot be contained in the subspace $u_{1}=0$, since this would yield a kernel to $\mathcal{L}$ in the exponentially weighted spaces $L_{\eta}^{2}$, which we excluded in Section 1.3.3 assuming generic interaction. Suppose therefore that there was an intersection outside of the $u_{2}$-subspace. We then can integrate the linearized evolution in the $u_{1}$-subspace and find the linearized $u_{1}$-component explicitly, independently of $u_{2}$. This $u_{1}$-component, proportional to $\mathrm{e}^{-\alpha x}$, however does not belong to either of the intersections. This establishes transversality and shows existence of a global center manifold. The smoothness of the manifold is a consequence of the strong spectral gap between the eigenvalues $-\alpha, \pm \beta$, and the remainder of the spectrum of the linearization at the origin, Hypothesis 1.2. This concludes the proof of the theorem.

We note that without the assumption of a strong spectral gap, the manifold would only be of class $C^{1, \iota}$, which would limit the subsequent analysis. Normal hyperbolicity implies that the the global center-manifold $\mathcal{M}_{\text {hom }}$ is tangent to the eigenspace associated with the eigenvalues $-\alpha, \pm \beta$, at the origin.

Corollary 5.3 The Turing patterns generated by the Turing spots, Lemma 4.2, possess smooth $N$-dimensional manifolds, each. 
Proof. The Turing patterns are hyperbolic outside of the homoclinic center manifold by reversibility and normal hyperbolicity of $\mathcal{M}_{\text {hom }}$. They therefore possess $N$-1-dimensional strong stable fibers, whose union is the desired stable manifold.

\subsection{Construction of a Poincaré map}

We can now construct a return map in the neighborhood of the homoclinic orbit. Consider therefore first the flow in the vicinity of the origin. We can parametrize the 3 -dimensional invariant manifold by coordinates $v_{\mathrm{ss}}, v_{\mathrm{uu}}$, and $v_{\mathrm{s}}$ in the linear eigenspace corresponding to the eigenvalues $-\beta, \beta,-\alpha$, respectively. The latter corresponds to the $u_{1}$-system and carries a trivial linear flow, since, in the normal form, nonlinearities always carry products of unstable and stable coordinates. We obtain the vector field induced on the center eigenspace by evaluating the vector field on the center manifold and then projecting onto the center direction. The dynamics can then be written in the form

$$
\begin{aligned}
\dot{v}_{\mathrm{ss}} & =-\beta v_{\mathrm{ss}}+\tilde{g}_{\mathrm{ss}}(v) \\
\dot{v}_{\mathrm{uu}} & =\beta v_{\mathrm{uu}}+\tilde{g}_{\mathrm{uu}}(v) \\
\dot{v}_{\mathrm{s}} & =-\alpha v_{\mathrm{s}} .
\end{aligned}
$$

Since after our normal form transformation the stable manifold is given by $v_{\mathrm{uu}}=0$, we conclude that this two-dimensional submanifold is invariant, which leads to $\tilde{g}_{\mathrm{uu}}(v)=\beta v_{\mathrm{uu}} g_{u u}\left(v_{\mathrm{uu}}, v_{\mathrm{ss}}\right)$. Together with reversibility, we therefore find the simplified normal form

$$
\begin{aligned}
\dot{v}_{\mathrm{ss}} & =-\beta\left(1+g\left(v_{\mathrm{ss}}, v_{\mathrm{uu}}\right)\right) v_{\mathrm{ss}} \\
\dot{v}_{\mathrm{uu}} & =\beta\left(1+g\left(v_{\mathrm{uu}}, v_{\mathrm{ss}}\right)\right) v_{\mathrm{uu}} \\
\dot{v}_{\mathrm{s}} & =-\alpha v_{\mathrm{s}},
\end{aligned}
$$

and $g(v)=\mathrm{O}(2)$. Moreover, since $g$ arises from terms of the form $u^{2 j} u^{2 j-1}$ in (5.2), $g$ is a constant of motion.

We next rescale so that the normalizing coordinate change covers the region $\left|v_{j}\right| \leq 1$ and introduce Poincaré sections $\Sigma^{\text {in }}=\left\{v_{\mathrm{ss}}=1\right\}$ and $\Sigma^{\text {out }}=\left\{v_{\mathrm{uu}}=1\right\}$, so that the homoclinic orbit intersects $\Sigma^{\text {in }}$ at $v_{\text {uu }}=v_{\mathrm{s}}=0$ and $\Sigma^{\text {out }}$ at $v_{\mathrm{ss}}=v_{\mathrm{s}}=0$; see Figure 5.2.

Using the time-reversed normalized flow, we can construct a first-hit map from $\Sigma^{\text {out }}$ to $\Sigma^{\text {in }}$. Since the arguments of $g$ are conserved quantities under the time evolution, we can write $g=g(v(0))=\mathrm{O}\left(|v(0)|^{2}\right)$. This gives the linear flow

$$
v_{\mathrm{SS}}(x)=v_{\mathrm{SS}}(0) \mathrm{e}^{\beta\left(1+g_{0}\left(v_{\mathrm{ss}}(0)\right)\right) x} .
$$

For the time of flight from $\Sigma^{\text {out }}$ to $\Sigma^{\text {in }}$, this gives

$$
T=-\frac{\log v_{\mathrm{sS}}^{\text {out }}}{\beta\left(1+g_{0}\left(v_{\mathrm{SS}}^{\text {out }}\right)\right)} .
$$

Substituting into the linear evolution for $v_{\mathrm{s}}$, we find

$$
v_{\mathrm{s}}^{\text {in }}=\left(v_{\mathrm{ss}}^{\text {out }}\right)^{-\gamma} S_{0}\left(v_{\mathrm{ss}}^{\text {out }}\right) v_{\mathrm{s}}^{\text {out }},
$$




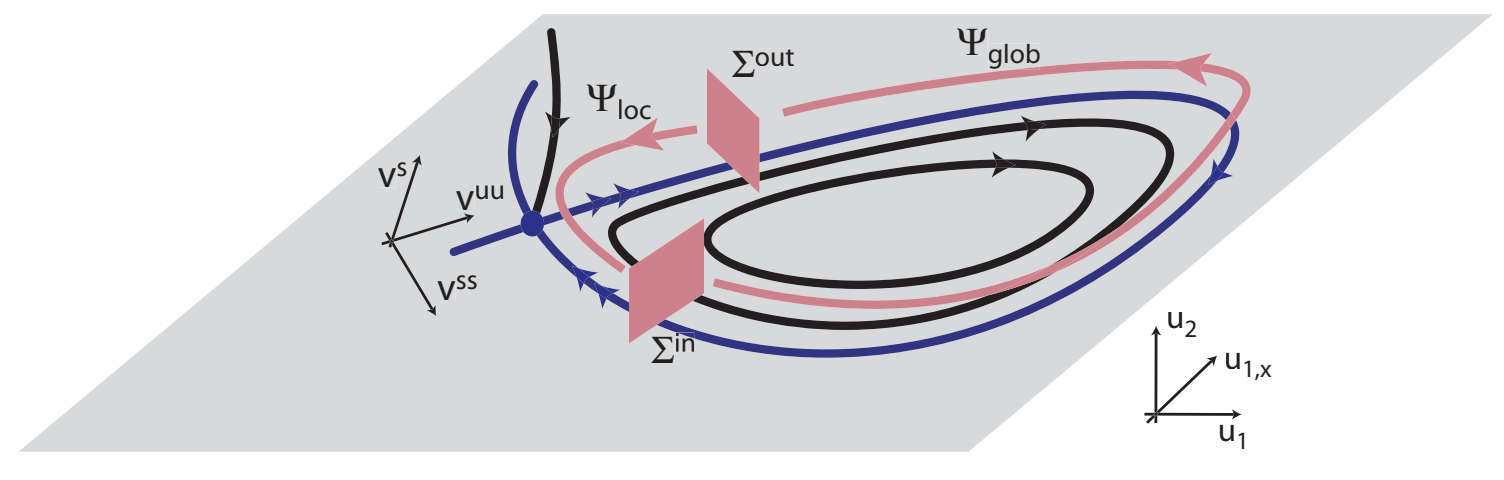

Figure 5.2: The flow near the homoclinic with Poincaré sections and first hit maps.

where $\gamma=\alpha / \beta<1$ and $S_{0}$ is $C^{2, \iota}$ in $v, \log v$ with $S_{0}(v)=1+\mathrm{O}\left(v^{2} \log v\right)$. Together with the trivial flow in the strong stable and unstable directions, we find the complete local first-hit map $\Psi_{\text {loc }}$,

$$
v_{\mathrm{uu}}^{\text {in }}=v_{\mathrm{ss}}^{\text {out }}, \quad v_{\mathrm{s}}^{\text {in }}=\left(v_{\mathrm{ss}}^{\text {out }}\right)^{-\gamma} S_{0}\left(v_{\mathrm{ss}}^{\text {out }}\right) v_{\mathrm{s}}^{\text {out }} .
$$

Composing the change of coordinates with the time-reversed global flow along the homoclinic, we also find a $C^{2}$-global first-hit map $\Psi_{\text {glob }}$ from $\Sigma^{\text {in }}$ to $\Sigma^{\text {out }}$, which can be expanded as

$$
\begin{aligned}
& v_{\mathrm{ss}}^{\text {out }}=v_{\mathrm{uu}}^{\text {in }}+\phi_{\mathrm{uu}}^{1} v_{\mathrm{s}}^{\text {in }}+R_{1}\left(v_{\mathrm{s}}^{\text {in }}, v_{\mathrm{uu}}^{\text {in }}\right), \\
& v_{\mathrm{s}}^{\text {out }}=\phi_{\mathrm{s}}^{1} v_{\mathrm{s}}^{\text {in }}+R_{2}\left(v_{\mathrm{s}}^{\text {in }}, v_{\mathrm{uu}}^{\text {in }}\right) .
\end{aligned}
$$

Here, the functions $R_{j}$ are of class $C^{2}$, given by the smoothness of the coordinate change, and satisfy

$$
R_{j}(0, \cdot)=0, \quad \partial_{1} R_{j}(0,0)=0 .
$$

In the first expansion of (5.5), we used the fact that the periodic orbits give fixed points in $v_{\mathrm{s}}=0$, and in the second equation we used the fact that the system with $v_{\mathrm{s}}=0$ is flow-invariant due to the skew-product structure.

Composing local and global first-hit maps, we find an expansion for the Poincaré map

$$
\Psi_{0}:=\Psi_{\text {loc }} \circ \Psi_{\text {glob }}: \Sigma_{\text {in }} \rightarrow \Sigma_{\text {in }},
$$

whenever the composition is defined.

For ease of notation, we henceforth write $p=v_{\mathrm{uu}}^{\text {in }}$, and $q=v_{\mathrm{s}}^{\text {in }}$, and find

$$
\begin{aligned}
& p^{\prime}=p+\phi_{1}^{\mathrm{uu}} q+R_{1}(q, p) \\
& q^{\prime}=\left(\phi_{1}^{\mathrm{s}} q+R_{2}(q, p)\right)\left(p+\phi_{1}^{\mathrm{uu}} q+R_{1}(q, p)\right)^{-\gamma} S_{0}\left(p+\phi_{1}^{\mathrm{uu}} q+R_{1}(q, p)\right),
\end{aligned}
$$

where $\gamma=\alpha / \beta<1$.

Proposition 5.4 Due to the assumptions on the repulsive nature of the boundary layer, Section 1.3.2, we have $\phi_{1}^{\mathrm{uu}}>0$. Moreover, $\phi_{1}^{\mathrm{s}}>0$.

We will relegate the proof of this proposition to Section 5.8. 


\subsection{Analysis of the Poincaré map — scaling and invariant manifolds}

For the remainder of the analysis, we rescale $p$ and $q$ to achieve that $\phi_{1}^{\mathrm{uu}}=1$ and $\phi_{1}^{\mathrm{s}}=1$, so that

$$
\begin{aligned}
& p^{\prime}=p+q+R_{1}(q, p) \\
& q^{\prime}=\left(q+R_{2}(q, p)\right)\left(p+q+R_{1}(q, p)\right)^{-\gamma} S_{0}\left(p+q+R_{1}(q, p)\right) .
\end{aligned}
$$

Note that the stable manifold of the origin is given by $p=0$. Under forward iteration with our map (which corresponds to backward transport with the flow), we find a preimage, $q \sim p^{1-\gamma}$. One can formally check that further backwards iterates retain this scaling, which motivates the blow-up type change of coordinates $q=p^{1-\gamma} \rho$. In the new coordinates $(p, \rho)$, we have

$$
\begin{aligned}
& p^{\prime}=p\left(1+\rho p^{-\gamma}\right)+R_{1}, \\
& \rho^{\prime}=\left(\rho p^{1-\gamma}+R_{2}\right)\left(p\left(1+\rho p^{-\gamma}\right)+R_{1}\right)^{-1} S_{0}\left(p\left(1+\rho p^{-\gamma}\right)+R_{1}\right),
\end{aligned}
$$

where now $R_{j}=R_{j}\left(\rho p^{1-\gamma}, p\right)$. We shall denote this map in the scaled variables by $\Psi$. Note that $\Psi$ has a continuous extension into $p=0$ near $\rho=1$. In fact, in this extension, $\rho=1, p=0$ is a fixed point. In the following, we will construct an unstable manifold for $\Psi$ near this fixed point. This unstable manifold will be our desired manifold, which under the forward iterates of the original map is invariant and contains points that converge to $p=0, \rho=1$, which in turn exhibit precisely the asymptotics claimed for Liesegang patterns.

We therefore set $\rho=1-\epsilon$ so that $\Psi$ becomes

$$
\begin{aligned}
& p^{\prime}=p^{1-\gamma}+p-\epsilon p^{1-\gamma}+R_{1} \\
& \epsilon^{\prime}=\left(p^{\gamma}+p^{\gamma-1}\left(R_{1}+R_{2}\right)\right)\left(1+p^{\gamma}+\epsilon+p^{\gamma-1} R_{1}\right)^{-1} S_{0}\left(p\left(1+(1-\epsilon) p^{-\gamma}\right)+R_{1}\right) .
\end{aligned}
$$

Here, $R_{j}=R_{j}\left((1-\epsilon) p^{1-\gamma}, p\right)$, and the expansions (5.6) hold. Of course, this map now possesses a fixed point in $p=0, \epsilon=0$. One can moreover collect leading order terms near this fixed point and find the map

$$
p^{\prime}=p^{1-\gamma}, \quad \epsilon^{\prime}=p^{\gamma} .
$$

This map is not differentiable, not even Lipshitz continuous at the origin, which precludes the simple application of a stable manifold theorem. The map does however possess the primary characteristics needed for the construction of an unstable manifold. For $p$ small, the linearization possesses approximate eigenspaces in the $p$-direction and in the $\epsilon$-direction, with expanding multiplier $\nu^{\mathrm{u}}=p^{-\gamma}$ and contracting multiplier $\nu^{\mathrm{s}}=0$. In fact, this map possesses

the explicit invariant manifold $\epsilon=p^{\gamma /(1-\gamma)}$, given as a graph over the unstable subspace $\epsilon=0$. Note however that this manifold is not smooth, not even Lipshitz when $\gamma<1 / 2$. In the following, we exploit expansion and contraction properties in order to construct the desired invariant manifold for perturbations of the leading-order part. The main result is as follows.

Proposition 5.5 The fixed point $p=0, \epsilon=0$ of the map $\Psi$ defined in (5.10) possesses a $C^{1}$ unstable manifold, given as a graph over the linear unstable subspace $\epsilon \equiv 0$.

The proof uses graph transform and follows the construction in [32, Ch. 5], with appropriate modifications. It will be split up into several lemmas. 


\subsection{Existence of unstable manifolds — a graph transform proof}

We will look for invariant manifolds in the form of continuous graphs $\epsilon=h(p), p \in[0, \delta]$, for some $\delta$ small enough. We equip the space of graphs with the supremum norm $|h|_{\infty}:=$ $\sup _{p}|h(p)|$. The actual construction of $h$ via a fixed point argument will be restricted to a subset of continuous graphs $\Sigma$, which is defined as

$$
\Sigma:=\left\{h ; h \in C^{0}([0, p]), h(0)=0,|h|_{\gamma} \leq M\right\},
$$

where $M$ is a fixed, large enough constant, and the weighted Lipshitz norm $|h|_{\gamma}$ is defined through

$$
|h|_{\gamma}:=\sup _{p_{1}>p_{2} \geq 0} \frac{\left|h\left(p_{1}\right)-h\left(p_{2}\right)\right|}{p_{1}-p_{2}} \cdot\left(p_{1}+p_{2}\right)^{\mu}, \quad \mu=\max \left\{0, \frac{1-2 \gamma}{1-\gamma}\right\} .
$$

In particular, $|\cdot|_{\gamma}$ denotes the usual Lipshitz norm for $\gamma \geq 1 / 2$ and allows for power growth of the Lipshitz constant near the origin for $\gamma<1 / 2$. The following lemma collects a few useful properties of $\Sigma$.

Lemma 5.6 Assume $0<\gamma<1$. Then we have

(i) $\Sigma$ is closed as a subset of $C^{0}$.

(ii) If $h \in \Sigma,|h(p)| \leq M p^{\gamma /(1-\gamma)}$.

(iii) $h_{0}(p):=p^{\gamma /(1-\gamma)} \in \Sigma$ with $M=1$.

(iv) If $f, g \in \Sigma$ for some $M$, then $f \cdot g \in \Sigma$ for some $M^{\prime}$.

(v) If $f \in \Sigma$ for some $M$ and $g$ is Lipshitz with $g(0)=0$, then $g \circ f, f \circ g \in \Sigma$ for some $M^{\prime}$.

Proof. The proof is trivial for $\gamma \geq 1 / 2$. The case of $\gamma<1 / 2$ is also straightforward. The first assertion follows immediately from the definition of $|\cdot|_{\gamma}$. The second assertion follows by setting $p_{2}=0$ in the definition of $|\cdot|_{\gamma}$. For (iii), we write

$$
h_{0}\left(p_{1}\right)-h_{0}\left(p_{2}\right)=\int_{0}^{1} h_{0}^{\prime}\left(\tau p_{1}+(1-\tau) p_{2}\right) \mathrm{d} \tau\left(p_{1}-p_{2}\right),
$$

so that

$$
\begin{aligned}
\frac{\left|h\left(p_{1}\right)-h\left(p_{2}\right)\right|}{p_{1}-p_{2}} \cdot\left(p_{1}+p_{2}\right)^{\frac{1-2 \gamma}{1-\gamma}} & =\frac{\gamma}{1-\gamma} \int_{0}^{1}\left(\tau p_{1}+(1-\tau) p_{2}\right)^{\frac{2 \gamma-1}{\gamma-1}} \mathrm{~d} \tau\left(p_{2}+p_{1}\right)^{\frac{1-2 \gamma}{\gamma-1}} \\
& =\frac{\gamma}{1-\gamma} \int_{0}^{1}\left(\frac{\tau+(1-\tau) \mu}{1+\mu}\right)^{\frac{2 \gamma-1}{\gamma-1}} \mathrm{~d} \tau \\
& \leq \frac{\gamma}{1-\gamma} \int_{0}^{1} \tau^{\frac{2 \gamma-1}{\gamma-1}} \\
& =1
\end{aligned}
$$

where we set $\mu=p_{2} / p_{1} \in[0,1)$, and used monotonicity in $\mu$ in the inequality. The final two assertions also follow directly from the definition. 
We can formally define a map $\Psi_{\#}$ on graphs, induced by $\Psi$, via

$$
\Psi(\operatorname{graph}(h)) \cap\{[0, \delta] \times \mathbb{R}\}=: \operatorname{graph}\left(\Psi_{\#} h\right) .
$$

For this to make sense, we need to show that the left-hand side is actually a graph over the domain $[0, \delta]$. Denote by $P_{1}$ the projection on the first, $p$-component, write $P_{2}=1-P_{1}$, and $P_{j} \Psi=\Psi_{j}, j=1,2$. Then the above equation translates into the pointwise equality

$$
\Psi(p, h(p))=\left(p^{\prime},\left(\Psi_{\#} h\right)\left(p^{\prime}\right)\right)
$$

The following lemma treats the first component of this equation,

$$
\Psi_{1}(p, h(p))=p^{\prime}
$$

Lemma 5.7 Given $h \in \Sigma$, the equation (5.14) can be inverted for every $p^{\prime} \in[0, \delta]$ with inverse $p=\varphi\left(p^{\prime}\right) \in[0, \delta]$. Moreover, $\varphi$ is of the form

$$
\varphi\left(p^{\prime}\right)=\left(p^{\prime}+r\left(p^{\prime}\right)\right)^{\frac{1}{1-\gamma}}
$$

and $r$ possesses a small Lipshitz constant. Moreover, given $h_{1}, h_{2} \in \Sigma$, we have that

$$
\left|r_{h_{1}}(p)-r_{h_{2}}(p)\right| \leq C\left(\min \left\{\left|\varphi_{h_{1}}(p)\right|,\left|\varphi_{h_{2}}(p)\right|\right\}\right)^{1-\gamma} \cdot\left|h_{1}-h_{2}\right|_{\infty},
$$

for some $C>0$.

Proof. We need to solve the equation

$$
p^{\prime}=p^{1-\gamma}+p-h(p) p^{1-\gamma}+R_{1}\left((1+h(p)) p^{1-\gamma}, p\right) .
$$

We therefore substitute $\sigma=p^{1-\gamma}$, which gives

$$
p^{\prime}=\sigma+\sigma^{\frac{1}{1-\gamma}}-h\left(\sigma^{\frac{1}{1-\gamma}}\right) \sigma+R_{1}\left(\left(1+h\left(\sigma^{\frac{1}{1-\gamma}}\right)\right) \sigma, \sigma^{\frac{1}{1-\gamma}}\right) .
$$

The right-hand side of this equation is of the form id $+T$. We claim that the Lipshitz constant of $T$ is small for $\delta$ sufficiently small. We can then easily solve using the contraction mapping principle and derive the conclusion of the lemma.

In order to estimate the Lipshitz constant of $T$, we need to estimate the three contributions. The first term, $\sigma^{\frac{1}{1-\gamma}}$ is clearly differentiable with small derivative in a vicinity of the origin, The second term can be estimated for $\sigma_{1}<\sigma_{2}$ as follows:

$$
\begin{aligned}
\left|\sigma_{1} h\left(\sigma_{1}^{\frac{1}{1-\gamma}}\right)-\sigma_{2} h\left(\sigma_{2}^{\frac{1}{1-\gamma}}\right)\right| & \leq\left|\sigma_{1}-\sigma_{2}\right||h|_{\infty}+\left(\sigma_{1}+\sigma_{2}\right) \operatorname{Lip} h \cdot\left|\sigma_{1}^{\frac{1}{1-\gamma}}-\sigma_{2}^{\frac{1}{1-\gamma}}\right| \\
& \leq\left(|h|_{\infty}+\left(\sigma_{1}+\sigma_{2}\right)|h|_{\gamma}\left(\sigma_{1}^{\frac{1}{1-\gamma}}+\sigma_{2}^{\frac{1}{1-\gamma}}\right)^{\frac{2 \gamma-1}{1-\gamma}}\left(\sigma_{1}+\sigma_{2}\right)^{\frac{1}{1-\gamma}}\right)\left|\sigma_{1}-\sigma_{2}\right| \\
& \leq\left(|h|_{\infty}+|h|_{\gamma}\left(\sigma_{1}+\sigma_{2}\right)^{\frac{\gamma}{\left(1-\gamma^{2}\right)}}\right)\left|\sigma_{1}-\sigma_{2}\right|,
\end{aligned}
$$

and again we find a small Lipshitz constant since $|h|_{\infty}$ is small for $\delta$ small, Lemma 5.6, (ii), and we can choose $\delta$ small while $|h|_{\gamma} \leq M$ is fixed, so that the second term in the last inequality is small. The estimates for the remainder $R_{1}$ are somewhat tedious but easier. 
It remains to estimate the dependence of $r$ on $h$. Subtracting the fixed point equations for $h_{1}$ and $h_{2}$ with equal $p$, we obtain

$$
0=\sigma_{1}-\sigma_{2}+\sigma_{1}^{\frac{1}{1-\gamma}}-\sigma_{2}^{\frac{1}{1-\gamma}}+h_{2}\left(\sigma_{2}^{\frac{1}{1-\gamma}}\right) \sigma_{2}-h_{1}\left(\sigma_{1}^{\frac{1}{1-\gamma}}\right) \sigma_{1}+\left.R_{1}\right|_{h_{1}, \sigma_{1}}-\left.R_{1}\right|_{h_{2}, \sigma_{2}} .
$$

From here, assuming $\sigma_{1}<\sigma_{2}$, we readily find the estimate

$$
\left|\sigma_{1}-\sigma_{2}\right| \leq \kappa_{1}\left|\sigma_{1}-\sigma_{2}\right|+c \sigma_{1} \cdot \delta h
$$

where $\kappa_{1} \ll 1, c>0$ is a constant that does not depend on $\delta$, and

$$
\delta h:=\left|h_{2}\left(\sigma_{2}^{\frac{1}{1-\gamma}}\right)-h_{1}\left(\sigma_{2}^{\frac{1}{1-\gamma}}\right)\right| .
$$

From here, we immediately obtain

$$
\left|r_{h_{1}}(p)-r_{h_{2}}(p)\right| \leq c\left|\sigma_{1}\right| \cdot\left|h_{1}-h_{2}\right|_{\infty},
$$

for some $c>0$, as claimed. Substituting $\varphi^{1-\gamma}$ for $\sigma$, this proves the lemma.

Remark 5.8 In fact, we found that the Lipshitz constant of $r$ is bounded by $p_{1}^{\gamma /(1-\gamma)}$.

We may now go ahead and define the graph transform

$$
\left(\Psi_{\#} h\right)(p)=\Psi_{2}(\varphi(p), h(\varphi(p))) .
$$

Lemma 5.9 For $M$ sufficiently large and $\delta$ sufficiently small, we have that $\Psi_{\#} h \in \Sigma$.

Proof. We can explicitly write $\Psi_{\#} h$ in the form

$$
\begin{aligned}
\left(\Psi_{\#} h\right)(p)= & \left(\varphi^{\gamma}(p)+\varphi^{\gamma-1}(p)\left(R_{1}+R_{2}\right)\right)\left(1+\varphi^{\gamma}(p)+h(\varphi(p))+\varphi^{\gamma-1}(p) R_{1}\right)^{-1} \\
& \cdot S_{0}\left(\varphi(p)\left(1+(1-h(\varphi(p))) p^{-\gamma}\right)+R_{1}\right)
\end{aligned}
$$

We will subsequently estimate the Lipshitz constants of all the terms in this expression.

Step 1: Estimates on $\left|\varphi^{\gamma}\right|_{\gamma}$

Since $\varphi^{\gamma}=(\mathrm{id}+r)^{\gamma /(1-\gamma)}$, with $r(0)=0$ and $r$ Lipshitz, this is a simple consequence of Lemma 5.7, (iii),(v).

Step 2: Estimates on $\left|\varphi^{\gamma} \cdot h \circ \varphi\right|_{\gamma}$

By Lemma 5.7, (iii),(v), it is sufficient to show that each factor belongs to $\Sigma$. The first factor belongs to $\Sigma$ as we saw in the previous step. Since $h$ belongs to $\Sigma$ and $\varphi$ is Lipshitz and vanishes at the origin, the second factor also belongs to $\Sigma$.

Step 3: Estimates on $\left|\varphi^{\gamma-1} R_{1}\left((1+(h \circ \varphi)) \varphi^{1-\gamma}, \cdot\right)\right|_{\gamma}$

It is only here that we actually need to exploit that $R_{1}$ is of class $C^{2}$. Since $R_{1}(0, p)=0$, we can write $R_{1}(q, p)=q r_{1}(q, p)$, with $r_{1}$ of class $C^{1}$, vanishing at the origin. We therefore find

$$
\varphi^{\gamma-1}(p) R_{1}\left(\left(1+(h(\varphi(p))) \varphi^{1-\gamma}(p), p\right)=(1+h(\varphi(p))) r_{1}\left((1+h(\varphi(p))) \varphi^{1-\gamma}(p), p\right) .\right.
$$


Since $r_{1}$ is of class $C^{1}$ and hence Lipshitz, it is sufficient to show that the argument belongs to $\Sigma$. For this, it is sufficient to show that $\varphi^{1-\gamma} h \circ \varphi$ belongs to $\Sigma$. Since $\varphi^{1-\gamma}$ is actually Lipshitz, hence belongs to $\Sigma$ for any $\gamma$, and $h \circ \varphi$ belongs to $\Sigma$ by Lemma 5.7, (v), the claim is a consequence of Lemma 5.7, (iv).

Since $S_{0}$ is $C^{1}$, it belongs to $\Sigma$ because of the above estimates for its arguments. This completes the proof of the lemma.

Remark 5.10 It is only in the estimates for the error terms that we actually use that the local Poincaré map is of class $C^{2}$. The rest of the analysis only requires $C^{1}$-smoothness. Inspecting the argument, one can presumably weaken the smoothness to a weighted Lipshitz estimate for the derivative. One may hope to be able to derive such estimates when replacing linearization with Ovsyannikov-Shilnikov coordinates [23, 31] and thus be able to drop the strong spectral gap assumption.

Lemma 5.11 Under the assumptions of Lemma 5.9, there is a $\kappa<1$ such that for all $h_{1}, h_{2} \in$ $\Sigma$, we have that

$$
\left|\Psi_{\#} h_{1}-\Psi_{\#} h_{2}\right|_{\infty} \leq \kappa\left|h_{1}-h_{2}\right| .
$$

In other words, $\Psi_{\#}$ defines a contraction mapping on the closed set $\Sigma$.

Proof. From Lemma 5.7, we have an estimate for the dependence of $\varphi$ on $h$. We can therefore estimate

$$
\begin{aligned}
\left|\varphi_{h_{1}}^{\gamma}-\varphi_{h_{2}}^{\gamma}\right| & \leq\left|\varphi_{h_{1}}^{2 \gamma-1}+\varphi_{h_{2}}^{2 \gamma-1}\right| \cdot\left|\varphi_{h_{1}}^{1-\gamma}-\varphi_{h_{2}}^{1-\gamma}\right| \\
& \leq C\left|\varphi_{h_{1}}^{2 \gamma-1}+\varphi_{h_{2}}^{2 \gamma-1}\right| \cdot\left|h_{1}-h_{2}\right|_{\infty} \min \left\{\varphi_{h_{1}}^{1-\gamma}, \varphi_{h_{2}}^{1-\gamma}\right\} \\
& \leq c\left|h_{1}-h_{2}\right|_{\infty},
\end{aligned}
$$

Similarly, one derives estimates for the terms involving $h \circ \varphi$.

Proof. [Proposition 5.5] Lemma 5.11 establishes the existence of an invariant manifold as a locally Lipshitz graph. In fact the manifold is $C^{1}$, with estimates on the derivative $\left|h^{\prime}\right| \leq$ $M p^{(1-2 \gamma) /(1-\gamma)}$ for $\gamma<1 / 2$ and uniform bounds for $\gamma \geq 1 / 2$. The proof of this fact follows almost verbatim the exposition in [32, Ch. 5.II] and will be omitted, here. This then concludes the proof of Proposition 5.5.

\subsection{Proof of Theorem 3}

We can now conclude the proof of our main geometric result. The invariant manifold that we constructed as a fixed point of $\Psi_{\#}$, and its orbit under the continuous time- $x$ evolution lie inside the locally invariant homoclinic center-manifold $\mathcal{M}_{\text {hom }}$, Proposition 5.2. The union of its strong stable fibers forms an $N$-dimensional $C^{1}$-manifold of solutions which converge to the homoclinic orbit as a set, which establishes the existence of the stable manifold of the homoclinic, Theorem 3. We can derive asymptotics of solutions inside this manifold as follows. 
Points in the unstable manifold converge to $p=0, \rho=1$ faster than exponentially. Since the distance between spikes is given by $T=-\frac{1}{\beta} \log p+\mathrm{O}(1)$, and $q=\mathrm{e}^{-\alpha x}$, we have that $q_{n+1} / q_{n} \sim C p_{n}^{\gamma}$. Since in particular, $\rho \rightarrow 1$, we have $q p^{\gamma-1} \rightarrow 1$, which gives $p_{n+1}^{\gamma-1} / p_{n}^{-1} \rightarrow C$, or

$$
p_{n+1}=C p_{n}^{\frac{1}{1-\gamma}}\left(1+\mathrm{o}_{n}(1)\right)
$$

and

$$
T_{n+1}=\frac{1}{1-\gamma} T_{n}+\mathrm{O}(1)=\frac{\beta}{\beta-\alpha} T_{n}+\mathrm{O}(1)
$$

This establishes the asymptotics as stated in Theorem 3 .

The $n$-spot manifolds in Theorem 3 are backward iterates of the stable manifold of the origin. The first backward iterate is obtained by iterating the stable manifold $p=0$ forward with $\Psi$, which gives $q \sim p^{1-\gamma}$ or $\epsilon \equiv 0$; see (5.8), (5.10). In particular, the backward iterate defines the trivial graph $h \equiv 0 \in \Sigma$, which converges towards the fixed point of the graph transform under forward iteration. This establishes the convergence of the $n$-spot manifolds to the Liesegang manifold.

\subsection{Glide-interaction balance in the spatial Poincaré map}

In this section, we prove Proposition 5.4. We first notice that the sign of $\phi_{1}^{\mathrm{s}}$ is positive since $q=0$ is invariant under the global flow and the sign of $q$ alias $v_{\mathrm{u}}$ is therefore preserved in the Poincaré map. We need to relate the sign of $\phi_{1}^{\text {uu }}$ to interaction of Turing spots and glide forces. We restrict our attention to solutions of the linearized equation that lie in the center bundle, that is, the tangent bundle of the homoclinic center manifold along the homoclinic orbit. Inside the center bundle, there is a two-dimensional subspace of solutions that are bounded as $x \rightarrow \infty$. The derivative of the homoclinic orbit gives one solution inside of this subspace. Another solution is found by solving the differential equation

$$
D u_{2}^{\prime \prime}+\partial_{2} f_{2}\left(0, u_{2}^{*}\right) u_{2}=\mathrm{e}^{-\alpha x} \partial_{1} f_{2}\left(0, u_{2}^{*}(x)\right) u_{1, \infty}^{*},
$$

so that $u_{2}$ is bounded for $x \rightarrow+\infty$ and grows at most with rate $\mathrm{e}^{-\beta x}$ for $x \rightarrow-\infty$. This solution $u_{2}^{\mathrm{bc}}$ is unique up to scalar multiples and addition of multiples of the derivative of the homoclinic.

The projection of this solution at time $-L, L \gg 1$, onto the unstable eigenspace gives the sign of the coefficient $\phi_{\mathrm{uu}}^{1}$. The projection is given by taking the scalar product with the bounded solution of the adjoint variational equation,

$$
\left\langle\left(\begin{array}{c}
u_{2}^{\mathrm{bc}} \\
\left(u_{2}^{\mathrm{bc}}\right)^{\prime}
\end{array}\right)\left(\begin{array}{c}
\varphi_{1} \\
\varphi_{2}
\end{array}\right)\right\rangle=\left\langle\left(\begin{array}{c}
u_{2}^{\mathrm{bc}} \\
\left(u_{2}^{\mathrm{bc}}\right)^{\prime}
\end{array}\right)\left(\begin{array}{c}
-(D \psi)^{\prime} \\
D \psi
\end{array}\right)\right\rangle=\left(D \psi,\left(u_{2}^{\mathrm{bc}}\right)^{\prime}\right)-\left((D \psi)^{\prime}, u_{2}^{\mathrm{bc}}\right),
$$

all evaluated at some point $x=L$, with $L$ large. Here, we also normalized the solution $\varphi=\left(\varphi_{1}, \varphi_{2}\right)^{T}$ to the first-order adjoint variational equation so that the second component is given by $D \psi$, with $\psi$ being the kernel to the second-order operator. 
On the other hand, we can compute the boundary force,

$$
\begin{aligned}
F_{\mathrm{bc}} & =\left(\psi(x), \mathrm{e}^{-\alpha x} \partial_{1} f_{2}\left(0, u_{2}^{*}(x)\right) u_{1, \infty}^{*},\right)_{L^{2}} \\
& =\left(\psi(x), D\left(u_{2}^{\mathrm{bc}}\right)^{\prime \prime}+\partial_{2} f_{2}\left(0, u_{2}^{*}\right) u_{2}^{\mathrm{bc}}\right)_{L^{2}} \\
& =\lim _{x \rightarrow-\infty}\left[-\left(D \psi,\left(u_{2}^{\mathrm{bc}}\right)^{\prime}\right)+\left(D(\psi)^{\prime}, u_{2}^{\mathrm{bc}}\right)\right]
\end{aligned}
$$

where we used partial integration and the fact that $e^{*}$ lies in the kernel of $\mathcal{L}^{*}$ in the last equality, so that only boundary terms are retained.

Together, (5.17) and (5.18) give us us the sign of $\phi_{\mathrm{uu}}^{1}$, up to the orientation of $\psi(-L)$ relative to $q^{\prime}(L)$. More precisely, $\phi_{\mathrm{uu}}^{1}>0$ if $F_{\mathrm{bc}}>0$ and $\left.\left(\psi(-L), q^{\prime}(L)\right)\right)<0$, or if $F_{\mathrm{bc}}<0$ and $\left.\left(\psi(-L), q^{\prime}(L)\right)\right)>0$. Expanding $\psi(-L)=-\psi_{\infty} \mathrm{e}^{-\beta L}$ and $q^{\prime}(L)=q_{\infty} \mathrm{e}^{-\beta L}$, this shows that the sign of $\Phi_{\mathrm{uu}}^{1}$ is opposite to the product $F_{\mathrm{bc}} \cdot F_{\mathrm{int}}$. This proves Proposition 5.4.

\subsection{Non-existence of Liesegang patterns}

In this section, we show that our two main ingredients, force balance and weakly decaying boundary layers, are both necessary for the existence of Liesegang patterns.

Proposition 5.12 For aligned forces, there are no Liesegang patterns with geometric spacing law.

Proof. The geometric spacing law requires that $T_{n+1} / T_{n} \rightarrow c>1$, which corresponds to $\left(p^{\prime}\right)^{c} / p \rightarrow 1$ for backwards iterates of our map $\Psi$ in (5.7). Since however $p^{\prime}=p+\phi_{\mathrm{uu}}^{1} q+R_{1}$, this is impossible for $\phi_{\mathrm{uu}}^{1}<0$ and $q$ small. Indeed, for $n$ large we would find $p^{\prime} \gg p$ and therefore the contradiction $0<p^{\prime}-p=-q+R_{1}<0$.

Proposition 5.13 For strongly localized boundary layers, $\beta<\alpha$, there are no Liesegang patterns.

Proof. In this case, there exists a normally hyperbolic center manifold $[13,30,25]$, which is two-dimensional and the maximal invariant set consists of only the homoclinic and the periodic orbits. A trajectory approaches the invariant manifold along strong stable foliations [13], so that it either follows a periodic orbit with asymptotic phase, or it follows the homoclinic with asymptotic phase. Since the homoclinic converges to the origin, any trajectory converging to the homoclinic would also converge to the origin, which excludes Liesegang patterns.

We also remark that in the absence of a skew-product structure, one would expect a minimal decay rate for the homoclinic for an open, dense set of reaction-diffusion systems. Again, one would typically find a normally hyperbolic invariant manifold containing only the homoclinic and periodic orbits, which exclude Liesegang and multi-spot patterns. 


\section{Examples}

\subsection{A scalar model}

We start with a scalar (unrealistic) model for both reaction and precipitation,

$$
\begin{aligned}
& u_{1, t}=u_{1, x x}-\alpha^{2} u_{1} \\
& u_{2, t}=u_{2, x x}-\beta^{2} u_{2}+u_{2}^{2}+u_{1}
\end{aligned}
$$

One readily checks that all the assumptions of our main theorem are satisfied in this system: the first component exhibits boundary layers with decay rate $\alpha$ for Dirichlet boundary conditions $u_{1}(x=0)=b_{0}, u_{1}^{*}(x)=b_{0} \mathrm{e}^{-\alpha x}$, so that $u_{1, \infty}^{*}=b_{0}$. The second component exhibits localized spots $u_{2}^{*}(x)>0$, with decay rate $\beta$. Since the linearization is self-adjoint, we have that $\psi(x)=u_{2, x}^{*}(x) /\left|u_{2, x}^{*}\right|^{2}$. Since $\psi(x)=-\psi(-x)>0$ for $x<0$, we have $\operatorname{sign} F_{\mathrm{bc}}=\operatorname{sign} b_{0}$. Similarly, $F_{\text {int }}<0$ since $D=$ id is scalar and the linearization is self-adjoint, so that there exist Liesegang patterns for $b_{0}>0$. We notice that all Liesegang patterns in this example are strongly unstable since individual spots are unstable. This instability is weak when spots are composed of widely separated layers, for instance in

$$
u_{2, t}=u_{2, x x}-\beta^{2} u_{2}\left(1-u_{2}\right)\left(u_{2}-\frac{1}{2}+\delta\right)+u_{1},
$$

with $\delta>0$ small.

\subsection{Boundary layers in $A+B \rightarrow C$}

Moving towards more realistic models, we discuss boundary layers in the specific bimolecular reaction $A+B \rightarrow C$, modeled by

$$
a_{t}=d_{a} a_{x x}-a b, \quad b_{t}=d_{b} b_{x x}-a b .
$$

The system possesses equilibria $b \equiv b_{*}, a \equiv 0$ and $a \equiv a_{*}, b \equiv 0$. The stationary system is

$$
a_{x}=a_{1}, a_{1, x}=d_{a}^{-1} a b, \quad b_{x}=b_{1}, b_{1, x}=d_{b}^{-1} a b .
$$

Linearization in $a=a_{*}, b=0$ gives $a_{x x}=\left(d_{a}^{-1} a_{*}\right) b, b_{x x}=\left(d_{b}^{-1} a_{*}\right) b$, with eigenvalues $\alpha_{1}^{2}=0$ and $\alpha_{2}^{2}=d_{b}^{-1} a_{*}$. The family of equilibria, parametrized by $a_{*} \neq 0$, therefore possesses a two-dimensional stable manifold, foliated by the strong stable manifolds of each individual equilibrium. One can now find boundary layers by transverse intersections of the boundary conditions $\mathcal{B}_{1}$ with this stable manifold. We note that in this case, the boundary layer actually selects an equilibrium $a_{*}$. Of course, a trivial change of coordinates $a_{\text {new }} \mid:=a-a_{*}$ recovers the asymptotic zero equilibrium state.

Conserved quantities of the form $\sum_{j} \kappa_{j} u^{j}=0$, here $a-b=0$, are typical in closed reactors due to stoichiometric constraints. The present example illustrates that our results carry over to systems with conserved quantities, in the present case $a-b$, although assumptions on Fredholm properties of the linearization and hyperbolicity in the spatial dynamics are violated: one can 
simply restrict the spatial dynamics to level sets of the conserved quantities and recover hyperbolicity and transversality on these level sets.

In our specific example, one can actually give somewhat more information on the boundary layer. Subtracting the two systems gives $\left(d_{a} a-d_{b} b\right)_{x x}=0$, so that for bounded solutions $d_{a} a=d_{b} b+\mu$ for some constant $\mu$. The equation for $b$ then becomes $b_{x x}=d_{a}^{-1}\left(b+d_{b}^{-1} \mu\right) b$, an integrable planar Hamiltonian system. If we focus on solutions converging to $b=0$, we have $\mu=d_{a} a_{*}>0$, and there is a unique positive solution $b(x)>0, b^{\prime}(x)<0, b \rightarrow 0$ for $x \rightarrow \infty$. Of course, $a=d_{a}^{-1} d_{b} b+a_{*}$ is then also positive.

One can also find boundary layers in the case when $a_{*}=b_{*}=0$, that is, when both reactants are localized near the boundary. The boundary-layer system then simply becomes $b_{x x}=d_{a}^{-1} b^{2}$, with solution $b(x)=6 d_{a} /\left(x-x_{0}\right)^{2}, a(x)=6 d_{b} /\left(x-x_{0}\right)^{2}$, for some $x_{0}>0$ depending on the boundary conditions. However, our results on Liesegang patterns do not cover this case of an algebraically decaying boundary layer.

\subsection{Spikes and layers in precipitation}

Precipitation is often modeled as a conversion process, where the sum of concentrations of reaction product and precipitate is conserved. This leads to systems for $u_{2}=(c, e)$ of the form

$$
c_{t}=d_{c} c_{x x}-g(c, e)+a b, \quad e_{t}=d_{e} e_{x x}+g(c, e),
$$

where typically $d_{e} \ll d_{c}$. Again, stationary profiles at $a b=0$ can be found "explicitly" after eliminating one variable using $d_{c} c+d_{e} e \equiv \mu$, which leads to

$$
d_{c} c_{x x}-g\left(c, \frac{\mu-d_{c} c}{d_{e}}\right)=0
$$

Turing spots, or precipitation spikes, are here a common phenomenon for non-monotone, multistable nonlinearities $g_{0}(c):=g\left(c, \frac{\mu-d_{c} c}{d_{e}}\right)$. A typical model for precipitation would include some type of threshold kinetics for $c$, in its simplest form given by $g(c, e)=c(c-1)\left(c-c_{\mathrm{th}}(e)\right)$, with threshold value $c_{\mathrm{th}}(e) \in(0,1)$ and increasing with $e$. Both $c=1$, undepleted reaction product, and $c=0$, completely precipitated reaction product, are stable states. For fixed $e$, precipitation spreads when $c_{\mathrm{th}}>1 / 2$. Since precipitation is enhanced by the presence of $e$, we assume $c_{\mathrm{th}}$ is increasing with $e$. The steady-state problem for $c$ becomes

$$
d_{c} c_{x x}+g_{0}(c)=0, \quad g_{0}(c)=c(1-c)\left(c-c_{\mathrm{th}}\left(\frac{\mu-d_{c} c}{d_{e}}\right)\right) .
$$

This system is integrable and straightforward to analyze using phase plane methods. It possesses an energy $\int\left(c_{x}^{2}+2 G_{0}(c)\right)$, where $G_{0}^{\prime}=-g_{0}$. It possesses three equilibria $c=0<c_{*}<1$. This can be readily seen solving the fixed point equation $c=c_{\text {th }}$ using monotonicity of $c_{\text {th }}$ and the fact that $c_{\mathrm{th}} \in(0,1)$. The outer equilibria $c=0,1$ are saddles and there are homoclinic orbits (alias Turing spots) to $c=0$ if $G_{0}(0)>G_{0}(1)$ or to $c=1$ if $G_{0}(0)<G_{0}(1)$. For $G_{0}(0)=G_{0}(1)$, there is a stationary interface separating $c=0$ from $c=1$. Determining stability and interaction properties of spikes in the system (6.4) appears to be an open question. 
Simpler examples of localized spots can of course be found in phenomenological models of precipitation such as the Cahn-Hilliard equation [6] $u_{2, t}=-\partial_{x x}\left(\partial_{x x} u_{2}+u_{2}-u_{2}^{3}+a b\right)$, which leads to the stationary problem $u_{2, x x}+u_{2}-u_{2}^{3}=\mu$, for spot solutions at $a b=0$.

\section{$7 \quad$ Summary and outlook}

We presented a conceptual framework for the analysis of stationary Liesegang patterns in reaction-diffusion systems. First ingredient are the localized precipitation zones, which correspond to localized spike-type solutions in an idealized unbounded system. We argue that in a reflection-symmetric system, such localized solutions typically simply repel or attract each other so that stationary Liesegang patterns are not possible in a generic reaction-diffusion system. We then argue that a skew-product structure caused by an irreversible chemical reaction can account for Liesegang patterns in an open class of reaction-diffusion systems and boundary conditions. The skew-product structure allows for a slowly decaying boundary layer in the first reaction, which then acts as a parameter ramp for the strongly localized precipitation spikes. Existence of a precipitation spike, a slowly decaying boundary layer, together with a sign condition on boundary forces versus interaction forces are then shown to be sufficient (and almost necessary) ingredients for the robust existence of Liesegang patterns. The proofs exploit dynamical systems methods for the interaction of exponentially decaying tails in boundary layers and precipitation spikes.

There are a number of open problems emerging from this conceptual approach. On the technical side, one would like to remove Hypothesis 1.2, at least partly. An interesting dynamical question arises when one investigates the limit of asymptotic Turing patterns as $k \rightarrow 0$ : it appears plausible, also from the simple mechanistic considerations in Section 2, that the stable manifolds of the periodic orbits converge as smooth manifolds to the Liesgang manifold. As a consequence one would find that robust Liesegang patterns enforce the existence of a family of asymptotic Turing patterns with arbitrary large wavelength. More generally, one might also be able to exclude the existence of solutions other than the family of multi-spots, the Liesegang pattern, and the asymptotic Turing patterns.

An interesting particular case is when the Turing spot is actually formed by two layers, that is, when the homoclinic orbit that represents the Turing spot in the $u_{2}$ spatial dynamics is actually a heteroclinic loop. An example is the system (6.5) when $G_{0}(0)=G_{0}(1)$, or the Cahn-Hilliard system with mass $\int u=0$. One can then extend the present analysis and attempt to recover spatial scaling laws predicted by the formal mechanistic model. One would in particular wish to recover a spatial scaling law for the width of the precipitation spikes.

Beyond the mere existence of Liesegang patterns, the main open questions that arise are concerned with the temporal dynamics of Liesegang patterns. One can envision the formation as a diffusive front establishes the boundary layer while moving through $x>0$. A stability analysis would at first involve the interaction of Turing spots, with likely slow instabilities such as in coarsening processes in Allen-Cahn or Cahn-Hilliard equations for $F_{\text {int }}<0$. In the Allen-Cahn equation, localized perturbations would then trigger a slow moving coarsening 
front [27], with speed exponentially slow in the distance between spots. More generally, there appears to be little understanding of pattern formation in systems with conserved quantities such as in the precipitation kinetics, and a more systematic analysis of coherent structures in such systems will be subject of future work.

\section{References}

[1] T. Antal, I. Bena, M. Droz, K. Martens, and Z. Rácz. Guiding fields for phase separation: Controlling Liesegang patterns. Phys. Rev. E 76 (2007), 046203.

[2] V. I. Arnold and M. B. Sevryuk. Oscillations and bifurcations in reversible systems. In: R. Z. Sagdeev, Editor, Nonlinear Phenomena in Plasma Physics and Hydrodynamics, Mir, Moscow (1986), pp. 31-64.

[3] G.R. Belitskii. Functional equations and conjugacy of local diffeomorphisms of a finite smoothness class. Funt. Anal. Appl. 7, 268-277.

[4] Y. Brechet and J.S. Kirkady. Contribution to the theory of diffusion-reaction controlled Liesegang patterns. J. Chem. Phys. 90 (1989), 1499.

[5] J. Cartwright, J.M. Garca-Ruiz, and A. I. Villacampa. Pattern formation in crystal growth: Liesegang rings. Computer Phys. Comm. 121-122 (1999), 411-413

[6] M. Droz. Recent Theoretical Developments on the Formation of Liesegang Patterns. J. Stat. Physics 101 (2000), 509-519.

[7] J. George and G. Varghese. Intermediate colloidal formation and the varying width of periodic precipitation bands in reaction-diffusion systems. J. Colloid Interface Sci. 282 (2005), 397-402.

[8] H.K. Henisch. Periodic Precipitation. Pergamon Press, Oxford, 1991.

[9] M. Hildebrand, A.S. Mikhailov, and G. Ertl. Nonequilibrium stationary microstructures in surface chemical reactions. Phys. Rev. E 58 (1998), 5483.

[10] D. Hilhorst, R. van der Hout, M. Mimura, and I. Ohnishi. Fast reaction limits and Liesegang bands. Free boundary problems, 241-250, Internat. Ser. Numer. Math., 154, Birkhuser, Basel, 2007.

[11] D. Hilhorst, R. van der Hout, and L.A. Peletier. Diffusion in the presence of fast reaction: the case of a general monotone reaction term. J. Math. Sci. Univ. Tokyo 4 (1997), 469517.

[12] D. Hilhorst, R. van der Hout, and L.A. Peletier. Nonlinear diffusion in the presence of fast reaction. Nonlinear Anal. 41 (2000), 803-823.

[13] A.J. Homburg. Global aspects of homoclinic bifurcations of vector fields. Memoirs of the A.M.S. 578, (1996). 
[14] J.B. Keller. Liesegang rings and a theory of fast reaction and slow diffusion. Dynamics and modelling of reactive systems (Proc. Adv. Sem., Math. Res. Center, Univ. Wisconsin, Madison, Wis., 1979), pp. 211-224, Publ. Math. Res. Center Univ. Wisconsin, 44, Academic Press, New York-London, 1980.

[15] J.B. Keller and S. Rubinow. Recurrent precipitation and Liesegang rings. J. Chem. Phys. 74 (1981), 5000-5007.

[16] H.-J. Krug, H. Brandstdter, and K. Jacob. Morphological instabilities in pattern formation by precipitation and crystallization processes. Int. J. Earth Sci. 85 (1996), 19-28.

[17] J. Lamb and J. Roberts. Time-reversal symmetry in dynamical systems: a survey. Timereversal symmetry in dynamical systems (Coventry, 1996). Phys. D 112 (1998), 1-39.

[18] M.I. Lebedeva, D.G. Vlachos, and M. Tsapatis. Bifurcation analysis of Liesegang ring pattern formation. Phys. Rev. Lett. 92 (2004), 088301.

[19] R. E. Liesegang. Über einige Eigenschaften von Gallerten. Naturwiss. Wochenschr. 11 (1896), 353-362.

[20] R. Matalon and A. Packter. The Liesegang phenomenon. I. Sol protection and diffusion. J.Colloid Sci. 10 (1955), 46-62.

[21] I. Ohnishi and M. Mimura. A mathematical aspect for liesegang phenomena. In Proceedings of the Equadiff 11 (2005), pp. 343-352.

[22] L. Ortiz-Bobadilla, A. Ortiz-Rodrguez, and E. Rosales-Gonzlez. Remark on finitely smooth linearization of local families of hyperbolic vector fields with resonances of high order. Bol. Soc. Mat. Mexicana 6 (2000), 199-212.

[23] I. Ovsyannikov and L. Shilnikov. Systems with a homoclinic curve of a multidimensional saddle-focus, and spiral chaos. Math. USSR-Sb. 73 (1992), 415-443.

[24] Z. Rácz. Formation of Liesegang patterns. Physica A 274 (1999), 50-59.

[25] B. Sandstede. Center manifolds for homoclinic solutions. J. Dyn. Diff. Eqns. 12 (2000), 449-510.

[26] B. Sandstede and A. Scheel. On the stability of periodic travelling waves with large spatial period. J. Diff. Eqns. 172 (2001), 134-188.

[27] A. Scheel. Coarsening fronts. Arch. Rat. Mech. Anal. 181 (2006), 505-534.

[28] V. Samovol. Smooth equivalence and linearization of reversible systems. Math. Notes 70 (2001), 86-96.

[29] G. Sell. Smooth linearization near a fixed point. Amer. J. Math., 107 (1985), 1035-1091

[30] M. Shashkov and D. Turaev. An existence theorem of smooth nonlocal center manifolds for systems close to a system with a homoclinic loop. J. Nonlinear Sci. 9 (1999), 525-573. 
[31] L. Shilnikov, A. Shilnikov, D. Turaev, and L. Chua. Methods of qualitative theory in nonlinear dynamics. Part II. World Sci. Ser. Nonl. Sci. Series A, 5, World Scientific, River Edge, NJ, 2001.

[32] M. Shub. Global stability of dynamical systems. Springer Verlag, 1987.

[33] M. Tsapatis, D. Vlachos, S. Kim, H. Ramanan, and G. Gavalas. Spontaneous formation of periodically patterned deposits by chemical vapor deposition. J. Am. Chem. Soc. 122 (2000), 12864-12865.

[34] A. Vanderbauwhede and B. Fiedler. Homoclinic period blow-up in reversible and conservative systems. Z. Angew. Math. Phys. 43 (1992), 292-318. 